\title{
SYNTHESIS AND INVESTIGATION OF MASS SPECTRA OF SOME NOVEL BENZIMIDAZOLE DERIVATIVES
}

\author{
Hassan K. Ibrahim $^{1 *}$, Sayed H. El-Tamany ${ }^{1}$, Reda F. El-Shaarawy ${ }^{1}$ Ibrahim M. El-Deen $^{2}$ \\ ${ }^{1}$ Faculty of Science, Suez Canal University, Ismailia, Egypt \\ ${ }^{2}$ Faculty of Education, Suez Canal University, Port Said, Egypt \\ hkhibrahim@hotmail.com
}

\begin{abstract}
2-Substituted benzimidazoles (3) and (4a-c) were prepared via condensation of ethyl 2-thionyl-pyruvate (1) and hydrazidoyl derivatives (2a-c) with $o$-phenylene diamine in acetic acid. Acetylation of compound (4a) with acetic anhydride yielded the corresponding $N$-acetyl derivative (5). Treatment of compound $\mathbf{4 b}$ with hydrazine hydrate gave the corresponding hydrazino derivative (6). Reaction of 2-[(2-thionyl)acetyl)] benzimidazole (3) with hydrazine hydrate gave the pyrazolyl derivative (7) and hydrazino derivative (8). Alkylation of compound 3 with alkyl halide gave the corresponding 2,3-disubstituled benzimidazoles (9a, b). Pyrrolo[1,2-a] benzimidazole derivatives 10 and 11 were prepared via cyclocondensation of compound $\mathbf{3}$ with acetic anhydride in presence of AcONa and phosphorus oxychloride.

The electron impact mass spectra of both of the above series of compounds have also been recorded and their fragmentation patterns are discussed.
\end{abstract}

Key words: mass spectrum; synthesis; and benzimidazoles

\section{СИНТЕЗА И ИСТРАЖУВАЊЕ НА МАСЕНИТЕ СПЕКТРИ НА НЕКОИ НОВИ БЕНЗИМИДАЗОЛНИ ДЕРИВАТИ}

Приготвени се 2-супституирани бензимидазоли (3) и (4a-c) со кондензација на етил-2-тионилпируват (1) и на деривати на хидразидоил 2a-c) со о-фенилен диамин во оцетна киселина. При ацетилирање на 4 a со анхидрид на оцетна киселина се добива соодветниот дериват на $N$-ацетил (5). Обработката на $4 \mathbf{b}$ со хидразинхидрат го дава соодветниот хиразинодериват (6). Реакцијата на 2-[(2-тионил)ацетил]бензимидазол (3) со хидразинхидрат ги дава дериватот на пиразолил (7) и хидразинодериватот (8). При алкилирање на 3 со алкилхалид се добиваат соодветните 2,3-дисупституирани бензимидазоли (9a, b). Дериватите на пироло[1,2а]бензимидазол 10 и 11 беа подготвени со циклокондензација на $\mathbf{3}$ со анхидрид на оцетна килсеина во присуство на AcONa и фосфороксихлорид.

Снимени се електронските масени спектри на двете наведени серии соединенија и дискутирани се нивните масени фрагментации.

Клучнин зборови: масени спектри; синтеза; бензимидазоли

\section{INTRODUCTION}

The benzimidazole ring is an important pharmacophore in modern drug discovery [1]. Benzimidazole derivatives exhibit significant activity against several viruses such as HIV [2,3], herpes (HSV-1) [4], RNA [5] and influenza [6].
Benzimidazoles are very important intermediates in organic reactions [7], therefore the preparation of benzimidazoles have gained considerable attention in recent years [8-10].

In a previous work, Mohy El-Deen et al [11, 12] reported the synthesis of 2-substituted quinoxaline from ethyl 2-thionylpyruvate (1) with $o$ - 
phenylene diamine in $4 \mathrm{~N}$ hydrochloric acid with ring cyclization with the removal of ethanol and water. This paper describes the synthesis of 2substituted benzimidazoles from condensation of ethyl 2-thionyl pyruvate and their derivatives (1) and (2a-c) with $o$-phenylene diamine in acetic acid. The electron impact (EI) ionization mass spectral fragmentation of the prepared compounds is also described.

\section{EXPERIMENTAL}

The melting points were determined in capillaries with a MEL-TEMP II Laboratory Devices, USA, and are uncorrected. Infrared spectra were recorded on Perkin-Elmer 337 Spectrophotometer using $\mathrm{KBr}$ wafers. Proton NMR spectra were obtained on a Varian EM 360 spectrometer using solution in hexadeuteriodimethyl sulfoxide with tetramethylsilane as the internal standard. Mass spectra were recorded on a VG Autspec GEIFAB ${ }^{+}$and a Hewlett Packard MS-Engine thermospray and ionization by electron impact at $70 \mathrm{eV}$. The accelerating voltage was $6 \mathrm{kV}$, the temperature of the source was $\sim 200{ }^{\circ} \mathrm{C}$, and the emission current $\sim 100 \mathrm{~mA}$. Micro-analysis was conducted using a Perkin-Elmer $2408 \mathrm{CHN}$ analyzer.

\section{Ethyl-2-thionylpyruvate (1)}

A mixture of 2-acetylthiophene $(0.01 \mathrm{~mol})$ and diethyl oxalate $(0.01 \mathrm{~mol})$ in $50 \mathrm{~mL}$ sodium methoxide solution $(0.005 \mathrm{~g} \mathrm{Na} / 25 \mathrm{~mL}$ methanol) was warmed for $20 \mathrm{~min}$, and then cooled. The solid that separated was washed with dilute hydrochloric acid and recrystallized from ethanol to give 1 as yellow crystals, m.p. $95{ }^{\circ} \mathrm{C}$, yield $82 \%, v_{\max }$ (KBr) $1745 \mathrm{~cm}^{-1}$ (CO of ester), $1643 \mathrm{~cm}^{-1}$ (CO of $\beta$-diketone). $\delta_{\mathrm{H}}\left(\mathrm{CDCl}_{3}\right): 1.30\left(\mathrm{t}, 3 \mathrm{H}, \mathrm{CH}_{3}\right), 3.31(\mathrm{~s}$, $\left.2 \mathrm{H}, \mathrm{COCH}_{2} \mathrm{CO}\right), 4.23\left(\mathrm{q}, 2 \mathrm{H}, \mathrm{OCH}_{2}\right)$ and 6.32 $7.50(\mathrm{~m}, 3 \mathrm{H}$, thiophene ring) ppm.

Anal. $\mathrm{C}_{10} \mathrm{H}_{10} \mathrm{O}_{4} \mathrm{~S}(226)$

Calcd: C 53.01; H 4.42; S $14.16 \%$

Found: C 52.89; H 4.24; S $14.00 \%$

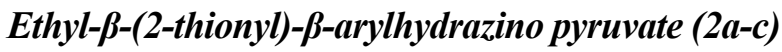

A solution of (1) $(0.01 \mathrm{~mol})$ in ethanol $(50$ $\mathrm{mL})$ was stirred with sodium acetate $(3 \mathrm{~g})$ and the mixture chilled in ice to $0-5{ }^{\circ} \mathrm{C}$. A cold aqueous solution $\left(0-5^{\circ} \mathrm{C}\right)$ of the diazonium salt $(0.01 \mathrm{~mol})$ was added dropwise with stirring for $45 \mathrm{~min}$. After addition the mixture was stirred for a further 30 $\min$ and then left for $2 \mathrm{~h}$ in a refrigerator. The precipitated products were collected, washed with water and recrystallized from ethanol to yield 2a-c.

Ethyl- $\beta$-(2-thionyl)- $\beta$-phenyl hydrazino pyruvate $(2 a)$, yield $68 \%, \mathrm{mp} .130^{\circ} \mathrm{C}, \boldsymbol{\nu}_{\max }(\mathrm{KBr})$ : $1739 \mathrm{~cm}^{-1}$ (CO of ester), $1635 \mathrm{~cm}^{-1}$ (CO of $\beta-$ diketone), $3401 \mathrm{~cm}^{-1}(\mathrm{NH}) . \delta_{\mathrm{H}}\left(\mathrm{CDCl}_{3}\right): 1.11(\mathrm{t}$, $\left.3 \mathrm{H}, \mathrm{CH}_{3}\right), 3.58\left(\mathrm{q}, 2 \mathrm{H}, \mathrm{OCH}_{2}\right), 7.24-8.10(\mathrm{~m}, 8 \mathrm{H}$, ArH and thiophene ring) and $11.10(\mathrm{~s}, 1 \mathrm{H}, \mathrm{NH})$ ppm.

Anal. $\mathrm{C}_{16} \mathrm{H}_{14} \mathrm{~N}_{2} \mathrm{O}_{4} \mathrm{~S}(330)$

Calcd: C 58.18; H 4.24; N 8.48; S 9.69\%

Found: C 58.02; H 4.07; N 8.22; S 9.36 \%

Ethyl- $\beta$-(2-thionyl)- $\beta$-(3-methylphenyl)hydrazino pyruvate (2b), yield $71 \%$, mp. $137^{\circ} \mathrm{C}, v_{\max }$ (KBr): $1732 \mathrm{~cm}^{-1}$ (CO of ester), $1632 \mathrm{~cm}^{-1}$ (CO of $\beta$-diketone), $3407(\mathrm{NH}) \mathrm{cm}^{-1} . \delta_{\mathrm{H}}\left(\mathrm{CDCl}_{3}\right): 1.20(\mathrm{t}$, $\left.3 \mathrm{H}, \mathrm{CH}_{3}\right), 2.32\left(\mathrm{~s}, 3 \mathrm{H}, \mathrm{CH}_{3}\right), 3.58\left(\mathrm{q}, 2 \mathrm{H}, \mathrm{OCH}_{2}\right)$, 6.81-7.98 (m, 7H, ArH and thiophene ring) and $11.10(\mathrm{~s}, 1 \mathrm{H}, \mathrm{NH}) \mathrm{ppm}$.

Anal. $\mathrm{C}_{17} \mathrm{H}_{16} \mathrm{~N}_{2} \mathrm{O}_{4} \mathrm{~S}(344)$

Calcd: C 59.30; H 4.65; N 8.14; S 9.30\%

Found: C 59.04; H 4.42; N 7.97; S 9.12\%

Ethyl- $\beta$-(2-thionyl)- $\beta$-(2-chlorophenyl) hydrazino pyruvate (2c), yield $72 \%$, m.p. $139^{\circ} \mathrm{C}, v_{\max }$ (KBr): $1738 \mathrm{~cm}^{-1}$ (CO of ester), $1635 \mathrm{~cm}^{-1}$ (CO of $\beta$-diketone), $3395 \mathrm{~cm}^{-1}(\mathrm{NH}), \delta_{\mathrm{H}}\left(\mathrm{CDCl}_{3}\right): 1.11(\mathrm{t}$, $\left.3 \mathrm{H}, \mathrm{CH}_{3}\right), 3.58$ (q, $\left.2 \mathrm{H}, \mathrm{OCH}_{2}\right), 7.24-8.10(\mathrm{~m}, 7 \mathrm{H}$, $\mathrm{ArH}$ and thiophene ring) and $11.10(\mathrm{~s}, 1 \mathrm{H}, \mathrm{NH})$ ppm.

Anal. $\mathrm{C}_{16} \mathrm{H}_{13} \mathrm{~N}_{2} \mathrm{ClO}_{4} \mathrm{~S}$ (364)

Calcd: C 52.73; H 3.57; N 7.69; Cl 9.62; S $8.79 \%$

Found: C 52.49; $\mathrm{H}$ 3.42; N 7.37; Cl 9.41; S $8.51 \%$

\section{2-(Substituted) acetylbenzimidazoles (3 and 4a-c)}

A mixture of 1 or $2 \mathrm{a}-\mathbf{c}(0.01 \mathrm{~mol})$ with $o^{-}$ phenylene diamine $(0.01 \mathrm{~mol})$ in acetic acid (5 $\mathrm{mL}$ ) was heated under reflux for $1 \mathrm{~h}$. The product obtained after cooling was collected by filtration, washed with ethanol, dried and purified by recrystallization with acetic acid to give $\mathbf{3}$ and $\mathbf{4 a - c}$.

$2-[(2-$ thionyl)acetyl)] benzimidazole (3), as yellow crystals, yield $67 \%$, m.p. $271{ }^{\circ} \mathrm{C}, v_{\max }$ 
(KBr): $3164 \mathrm{~cm}^{-1}(\mathrm{NH}), 1680 \mathrm{~cm}^{-1}(\mathrm{CO}$ of $\beta-$ diketone), $1620 \mathrm{~cm}^{-1}(\mathrm{C}=\mathrm{N}), 1603,1582 \mathrm{~cm}^{-1}$ $(\mathrm{C}=\mathrm{C}) . \delta_{\mathrm{H}}\left(\mathrm{DMSO}_{-} \mathrm{d}_{6}\right): 3.37\left(\mathrm{~s}, 2 \mathrm{H}, \mathrm{COCH}_{2} \mathrm{CO}\right)$, 6.7-7.98 (m, 7H, ArH and thiophene ring) and $12.06(\mathrm{~s}, 1 \mathrm{H}, \mathrm{NH}) \mathrm{ppm}$.

Anal. $\mathrm{C}_{14} \mathrm{H}_{10} \mathrm{~N}_{2} \mathrm{O}_{2} \mathrm{~S}(270)$

Calcd: C 62.22; H 3.70; N 10.37; S $11.85 \%$

Found: C 62.01; H 3.48; N 10.02; S $11.61 \%$

$2-[(\beta-2$ thionyl) $-\beta-($ phenyl $)$ hydrazino $]$ acetylbenzimidazole (4a) as orange crystals, yield $69 \%$, m.p. $240{ }^{\circ} \mathrm{C}$. $v_{\max }(\mathrm{KBr}): 3229,3187 \mathrm{~cm}^{-1}(\mathrm{NH})$, $1675 \mathrm{~cm}^{-1}$ (CO of $\beta$-diketone), $1628 \mathrm{~cm}^{-1}(\mathrm{C}=\mathrm{N})$, $1605,1588 \mathrm{~cm}^{-1}(\mathrm{C}=\mathrm{C}) . \delta_{\mathrm{H}}\left(\mathrm{DMSO}_{6}\right): 6.88-7.94$ (m, 12H, ArH and thiophene ring), $11.03(\mathrm{~s}, 1 \mathrm{H}$, $\mathrm{NNH})$ and $12.67(\mathrm{~s}, 1 \mathrm{H}, \mathrm{NH}) \mathrm{ppm}$.

Anal. $\mathrm{C}_{20} \mathrm{H}_{14} \mathrm{~N}_{4} \mathrm{O}_{2} \mathrm{~S}$ (374)

Calcd: C 64.17; H 3.74; N 14.97; S $8.55 \%$

Found: C 63.98; H 3.52; N 14.69; S 8.27 \%

$2-[\beta-(2$-thionyl)- $\beta$-(3-methylphenyl) hydrazino]-acetylbenzimidazole (4b) as orange crystals, yield $65 \%$, m.p. $160{ }^{\circ} \mathrm{C}$. $v_{\max }(\mathrm{KBr}): 3220,3185$ $\mathrm{cm}^{-1}(\mathrm{NH}), 1672(\mathrm{CO}$ of $\beta$-diketone), $1623(\mathrm{C}=\mathrm{N})$, $1606,1591 \mathrm{~cm}^{-1}(\mathrm{C}=\mathrm{C}) . \delta_{\mathrm{H}}\left(\mathrm{DMSO}-\mathrm{d}_{6}\right): 2.32(\mathrm{~s}$, $\left.3 \mathrm{H}, \mathrm{CH}_{3}\right), 6.88-7.94(\mathrm{~m}, 11 \mathrm{H}, \mathrm{ArH}$ and thiophene ring), $11.037(\mathrm{~s}, 1 \mathrm{H}, \mathrm{NNH})$ and $12.6(\mathrm{~s}, 1 \mathrm{H}, \mathrm{NH})$ ppm.

Anal. $\mathrm{C}_{21} \mathrm{H}_{16} \mathrm{~N}_{4} \mathrm{O}_{2} \mathrm{~S}(388)$

Calcd: C 64.95; H 4.12; N 14.43; S 8.25 \%

Found: C 64.67; H 4.01; N 14.20; S 8.02\%

$2-[\beta-(2$-thionyl)- $\beta$-(2-chlorophenyl) hydrazino]-acetylbenzimidazole $(\mathbf{4 c})$ as red crystals, yield $66 \%$, m.p: $182{ }^{\circ} \mathrm{C}$. $v_{\max }(\mathrm{KBr}): 3227,3183 \mathrm{~cm}^{-1}$ (NH), $1672 \mathrm{~cm}^{-1}$ (CO of $\beta$-diketone), $1623 \mathrm{~cm}^{-1}$ $(\mathrm{C}=\mathrm{N}), 1603,1589 \mathrm{~cm}^{-1}(\mathrm{C}=\mathrm{C}) . \delta_{\mathrm{H}}\left(\mathrm{DMSO}^{-\mathrm{d}_{6}}\right)$ : 6.96-7.7 (m, 11H, ArH and thiophene ring), 11.10 $(\mathrm{s}, 1 \mathrm{H}, \mathrm{NH})$ and $12.6(\mathrm{~s}, 1 \mathrm{H}, \mathrm{NH}) \mathrm{ppm}$.

Anal $\mathrm{C}_{20} \mathrm{H}_{13} \mathrm{~N}_{4} \mathrm{ClO}_{2} \mathrm{~S}$ (405.5)

Calcd: C 58.82; H 3.19; N 13.73; Cl 8.58; S $7.84 \%$

Found: C 58.52; H 3.03; N 13.42; Cl 8.29; S $7.61 \%$

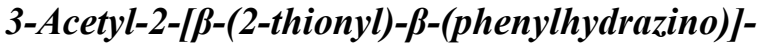 acetylbenzimidazole (5)}

A solution of $4 \mathbf{a}(0.01 \mathrm{~mol})$ in acetic anhydride $(30 \mathrm{~mL})$ was heated under reflux for $3 \mathrm{~h}$. The product formed after being cooled was filtered off, dried and purified by recrystallization with ethanol to give 5 as yellow crystals, yield $63 \%$, m.p. $145^{\circ} \mathrm{C} . v_{\max }(\mathrm{KBr}): 3185 \mathrm{~cm}^{-1}(\mathrm{NH}), 1685,1665$ $\mathrm{cm}^{-1}$ (CO of $\beta$-diketone and acetyl), $1628 \mathrm{~cm}^{-1}$ $(\mathrm{C}=\mathrm{N}), 1603,1589 \mathrm{~cm}^{-1}(\mathrm{C}=\mathrm{C}) . \delta_{\mathrm{H}}\left(\mathrm{DMSO}_{\mathrm{d}}\right)$ : $2.32\left(\mathrm{~s}, 3 \mathrm{H}, \mathrm{CH}_{3} \mathrm{CO}\right), 6.88-7.94(\mathrm{~m}, 12 \mathrm{H}, \mathrm{ArH}$ and thiophene ring) and $11.03(\mathrm{~s}, 1 \mathrm{H}, \mathrm{NH}) \mathrm{ppm}$.

Anal. $\mathrm{C}_{22} \mathrm{H}_{16} \mathrm{~N}_{4} \mathrm{O}_{3} \mathrm{~S}(416)$

Calcd: C 63.46; H 3.85; N 13.46; S 7.69 \%

Found: C 63.16; H 3.62; N 13.21; S $7.46 \%$

\section{Reaction of $4 b$ with hydrazine hydrate, formation of (6)}

A mixture of $4 \mathbf{b}(0.01 \mathrm{~mol})$ and hydrazine hydrate $(0.02 \mathrm{~mol})$ was fused on a hot plate for 2$3 \mathrm{~min}$, after which dimethyl formamide was add $(30 \mathrm{~mL})$. The mixture was heated under reflux for $2-3 \mathrm{~h}$, and then cooled and poured into water. The solid formed was filtered off, washed with water, dried, and recrystallized from ethanol to give $\mathbf{6}$ as yellow crystals, yield $63 \%$, m.p. $160{ }^{\circ} \mathrm{C} . v_{\max }$ (KBr): $3402,3122 \mathrm{~cm}^{-1}\left(\mathrm{NH}_{2}\right), 3281 \mathrm{~cm}^{-1}(\mathrm{NH})$, $1631 \mathrm{~cm}^{-1}(\mathrm{C}=\mathrm{N}), 1607,1591 \mathrm{~cm}^{-1}(\mathrm{C}=\mathrm{C})$. Mass spectrum, $m / z(\%): 416\left(\mathrm{M}^{+}, 7.10\right) 415\left(\mathrm{M}^{+}-1\right.$, 13.80), 257 (6.90), 256 (9.50), 196 (3.70), 195 (7.90) 185 (5.00), 176 (1.30), 162 (22.00), 161 (19.30), 160 (96.60), 159 (6.30), 149 (9.50), 143 (5.60), 139 (11.90), 133 (6.30), 132 (100), 131 (73.00), 122 (5.00), 120 (4.50), 117 (2.90), 116 (3.20), 111 (6.60), 105 (6.30), 104 (6.90), 103 (5.00), 102 (5.30), 92 (6.90), 91 (5.00), 90 (11.40) 85 (4.80), 83 (3.20), 77 (7.10), 76 (12.40), 66 (20.10), 65 (18.80), 64 (51.60), 63 (31.50), 62 (11.10), 52 (16.90) .

Anal. $\mathrm{C}_{21} \mathrm{H}_{20} \mathrm{~N}_{8} \mathrm{~S}(416)$

Calcd: C 60.58; H 4.81; N 26.92; S $7.69 \%$

Found: C 60.31; H 4.52; N 26.66; S $7.39 \%$

\section{2-[5-(2-thionyl) pyrazol-3-yl]benzimidazole (7)

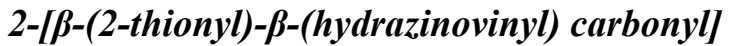 benzimidazole (8)}

A mixture of $3(0.01 \mathrm{~mol})$ and hydrazine hydrate $(0.01 \mathrm{~mol})$ in dimethyl formamide was heated under reflux for $2-3 \mathrm{~h}$. The reaction mixture was cooled and poured into water. The product formed was collected by filtration, washed with water, dried and dissolved in hot ethanol. The insoluble solid in hot ethanol was filtered off and 
purified by recrystallization with xylene to give $\mathbf{8}$. The filtrate was cooled and the solid formed was filtered off, dried and purified by recrystallization with ethanol to give 7, pale yellow crystals, yield $43 \%$, m.p. $180{ }^{\circ} \mathrm{C}$. $v_{\max }(\mathrm{KBr}): 3225 \mathrm{~cm}^{-1}(\mathrm{NH})$, $1630 \mathrm{~cm}^{-1}(\mathrm{C}=\mathrm{N}), 1606,1589 \mathrm{~cm}^{-1}(\mathrm{C}=\mathrm{C})$. Mass spectrum, $m / z(\%): 267\left(\mathrm{M}^{+}, 33.80\right) 183(5.80)$, 182 (37.20), 131 (28.80), 122 (17.30), 118 (17.50), 111 (30.00), 109 (38.80), 108 (77.90), 107 (22.50), 98 (22.50), 97 (26.30), 92 (27.50), 91 (33.80), 90 (52.50), 84 (38.80), 83 (35.00), 78 (43.80), 77 (78.80), 76 (40.60), 74 (17.50), 67 (36.20), 66 (32.20), 65 (92.50), 64 (56.30), 63 (72.50), 53 (66.30), 52 (100), 51 (78.80), 50 (73.80).

Anal. $\mathrm{C}_{14} \mathrm{H}_{10} \mathrm{~N}_{4} \mathrm{~S}(267)$

Calcd: C 62.92; H 3.75; N 20.97; S $11.98 \%$

Found: C 62.71; H 3.43; N 20.69; S $11.59 \%$

Compound (8) as yellow crystal, yield $33 \%$, m.p. $205^{\circ} \mathrm{C} . v_{\max }(\mathrm{KBr}): 3343,3105\left(\mathrm{NH}_{2}\right), 3253$ $\mathrm{cm}^{-1}(\mathrm{NH}), 1665 \mathrm{~cm}^{-1}(\mathrm{C}=\mathrm{O}), 1625 \mathrm{~cm}^{-1}(\mathrm{C}=\mathrm{N})$, $1604,1595 \mathrm{~cm}^{-1}(\mathrm{C}=\mathrm{C})$. Mass spectrum, $\mathrm{m} / z(\%)$ : $285\left(\mathrm{M}^{+}+1,7.60\right), 284\left(\mathrm{M}^{+}, 39.20\right), 256(22.80)$, 226 (3.80), 225 (22.80), 132 (17.70), 131 (29.10), 121 (29.10), 119 (25.30), 111 (19.00), 109 (30.60), 108 (100), 107 (17.70), 104 (24.10), 103 (26.60), 92 (39.20), 91 (27.80), 90 (15.20), 84 (32.40), 83 (15.20), 78 (39.20), 77 (25.80), 76 (29.10), 69 (45.60), 65 (24.10), 64 (34.20), 63 (29.10), 51 (30.40), 50 (43.00).

Anal. $\mathrm{C}_{14} \mathrm{H}_{12} \mathrm{~N}_{4} \mathrm{OS}(284)$

Calcd: C 59.15; H 4.23; N 19.72; S $11.27 \%$

Found: C 58.87; H 4.00; N 19.49; S $11.03 \%$

\section{3-Substituted-2-[6-(thionyl)acetyl]benzimidazoles $(9 a, b)$}

A mixture of $3(0.01 \mathrm{~mol})$ and alkyl halides (such as benzyl chloride and phenacyl bromide) $(0.01 \mathrm{~mol})$ in dimethyl formamide $(30 \mathrm{~mL})$ were heated under reflux for $4 \mathrm{~h}$. The reaction mixture was cooled and poured into water. The product formed was collected by filtration, washed with water, dried and purified by recrystallization with ethanol to give 9 .

3-Benzyl-2-[(2-thiony)l acetyl] benzimidazole (9a) as yellow crystals, yield $61 \%$, m.p. $197^{\circ} \mathrm{C}$. $v_{\max }(\mathrm{KBr}): 1675 \mathrm{~cm}^{-1}$ (CO of $\beta$-diketone), 1624 $\mathrm{cm}^{-1}(\mathrm{C}=\mathrm{N}), 1605,1589 \mathrm{~cm}^{-1}(\mathrm{C}=\mathrm{C}) . \delta_{\mathrm{H}}(\mathrm{DMSO}-$ $\left.\mathrm{d}_{6}\right): 3.428\left(\mathrm{~s}, 2 \mathrm{H}, \mathrm{COCH}_{2} \mathrm{CO}\right), 5.93\left(\mathrm{~s}, 2 \mathrm{H}, \mathrm{CH}_{2}\right)$, and $6.7-7.98(\mathrm{~m}, 12 \mathrm{H}, \mathrm{ArH}$ and thiophene ring) ppm.
Anal. $\mathrm{C}_{21} \mathrm{H}_{16} \mathrm{~N}_{2} \mathrm{O}_{2} \mathrm{~S}(360)$

Calcd: C 69.81; H 4.43; N 7.75; S $8.86 \%$

Found:C 69.63; H 4.22; N 7.51; S $8.48 \%$

3-Benzylmethyl-2-[(2-thionyl) acetyl] benzimidazole (9b) as yellow crystals, yield $68 \%$, m.p. $240{ }^{\circ} \mathrm{C}, v_{\max }(\mathrm{KBr}): 1688 \mathrm{~cm}^{-1}$ (CO of ketone), $1671 \mathrm{~cm}^{-1}$ (CO of $\beta$-diketone) $1627 \mathrm{~cm}^{-1}(\mathrm{C}=\mathrm{N})$, $1605,1591 \mathrm{~cm}^{-1}(\mathrm{C}=\mathrm{C}) \cdot \delta_{\mathrm{H}}\left(\mathrm{DMSO}-\mathrm{d}_{6}\right): 3.428(\mathrm{~s}$, $\left.2 \mathrm{H}, \mathrm{COCH}_{2} \mathrm{CO}\right), 6.22\left(\mathrm{~s}, 2 \mathrm{H}, \mathrm{CH}_{2}\right)$, and 6.7-7.98 ( $\mathrm{m}, 12 \mathrm{H}, \mathrm{ArH}$ and thiophene ring) $\mathrm{ppm}$.

Anal. $\mathrm{C}_{22} \mathrm{H}_{16} \mathrm{~N}_{2} \mathrm{O}_{3} \mathrm{~S}(388)$

Calcd: C 68.04; H 4.12; N 7.22; S 8.25\%

Found:C 67.88; H 4.00; N 6.98; S 8.01\%

\section{2-Methyl-3-(2-thionyl)-4H-4-oxo-pyrrolo[1,2- aJbenzimidazole (10)}

A mixture of $3(0.01 \mathrm{~mol})$ and fused sodium acetate $(0.02 \mathrm{~mol})$ in acetic anhydride $(30 \mathrm{~mL})$ was heated under reflux for $4 \mathrm{~h}$. The reaction mixture was cooled and poured into water. The solid formed was collected by filtration, washed with water, dried and purified by recrystallization with acetic acid to give $\mathbf{1 0}$ as yellow crystals, m.p. 260 ${ }^{\circ} \mathrm{C} . v_{\max }(\mathrm{KBr}): 1685,1668 \mathrm{~cm}^{-1}(\mathrm{CO}), 1625 \mathrm{~cm}^{-1}$ $(\mathrm{C}=\mathrm{N}), 1603,1591 \mathrm{~cm}^{-1}(\mathrm{C}=\mathrm{C}) . \delta_{\mathrm{H}}\left(\mathrm{DMSO}^{-\mathrm{d}_{6}}\right)$ : $2.556\left(\mathrm{~s}, 3 \mathrm{H}, \mathrm{CH}_{3}\right)$, and 6.7-7.98 (m, 7H, ArH and thiophene ring) ppm.

Anal. $\mathrm{C}_{16} \mathrm{H}_{10} \mathrm{~N}_{2} \mathrm{O}_{2} \mathrm{~S}(294)$

Calcd: C 65.31; H 3.40; N 9.52; S $10.88 \%$

Found:C 65.00; H 3.13; N 9.25; S $10.63 \%$

\section{5-(2-thinyl)-3H-3-oxo-pyrrolo [1,2-a] benzimidazole (11)}

A solution of (3) $(0.01 \mathrm{~mol})$ in phosphorus oxychloride $(30 \mathrm{~mL})$ was heated on a water bath for 3-4 h. The reaction mixture was cooled and poured into water. The product obtained was filtered off, washed with water, dried and recrystallized from ethanol to give $\mathbf{1 1}$ as pale yellow crystals, yield $58 \%$, m.p. $190{ }^{\circ} \mathrm{C}$. $v_{\max }(\mathrm{KBr}): 1672 \mathrm{~cm}^{-1}$ $(\mathrm{C}=\mathrm{O}), 1627 \mathrm{~cm}^{-1}(\mathrm{C}=\mathrm{N}), 1605,1595 \mathrm{~cm}^{-1}(\mathrm{C}=\mathrm{C})$. $\delta_{\mathrm{H}}\left(\mathrm{DMSO}_{-} \mathrm{d}_{6}\right): 6.7-7.98(\mathrm{~m}, 8 \mathrm{H}, \mathrm{ArH}$, pyrrolo and thiophene ring) ppm.

Anal. $\mathrm{C}_{14} \mathrm{H}_{8} \mathrm{~N}_{2} \mathrm{OS}(252)$

Calcd: C 66.66; H 3.17; N 11.11; S $12.70 \%$

Found:C 66.36, H 3.02, N 10.98, S $12.42 \%$ 


\section{RESULTS AND DISCUSSION}

\section{Chemistry}

Ethyl 2-thionyl pyruvate (1) was prepared from 2-acetyl thiophene and diethyl oxalate in the presence of methoxide according to a published literature procedure [13].

The hydrazidoyl derivatives (2a-c) were prepared by coupling of (1) with diazotized amines, namely aniline, $m$-toludine and $o$-chloroaniline in ethanolic solution buffered with sodium acetate $[14,15]$.

Condensation of $\mathbf{1}$ and 2a-c with $o$-phenylene diamine in glacial acetic acid under reflux, gave the corresponding 2-[(2-thionyl)acetyl] benzimidazole (3) and 1-(1H-benzoimidazol-2-yl)-2-(2-(substituted phenyl) hydrazono)-3-(thiophen-2-yl) propan-1,3dione (4a-c), respectively. 1-(1-acetyl-benzoimidazol-2-yl)-2-(2-phenylhydrazono)-3-(thiophen-2-yl)propan-1,3-dione (5) was prepared by acetylation of $\mathbf{4 a}$ with acetic anhydride. The reaction of $\mathbf{4 b}$ with hydrazine hydrate in dimethyl formamide under reflux gave the corresponding hydrazino derivatives (6) (Scheme 1).

Condensation of 2-[2-thionyl acetyl] benzimidazole (3) with hydrazine hydrate in dimethyl formamide yielded the corresponding 2-(5-thionylpyrazol-3-yl)benzimidazole (7) and 2-[( $\beta-2$-thionyl- $\beta$-hydrazino) vinyl] carbonyl-benzimidazole (8). Treatment of compound $\mathbf{3}$ with alkyl halides such as benzyl chloride and phenacyl bromide in dimethyl formamide under reflux, yielded the corresponding 3-substituted-2-[(2-thionyl)acetyl]benzimidazoles (9a, 9b)

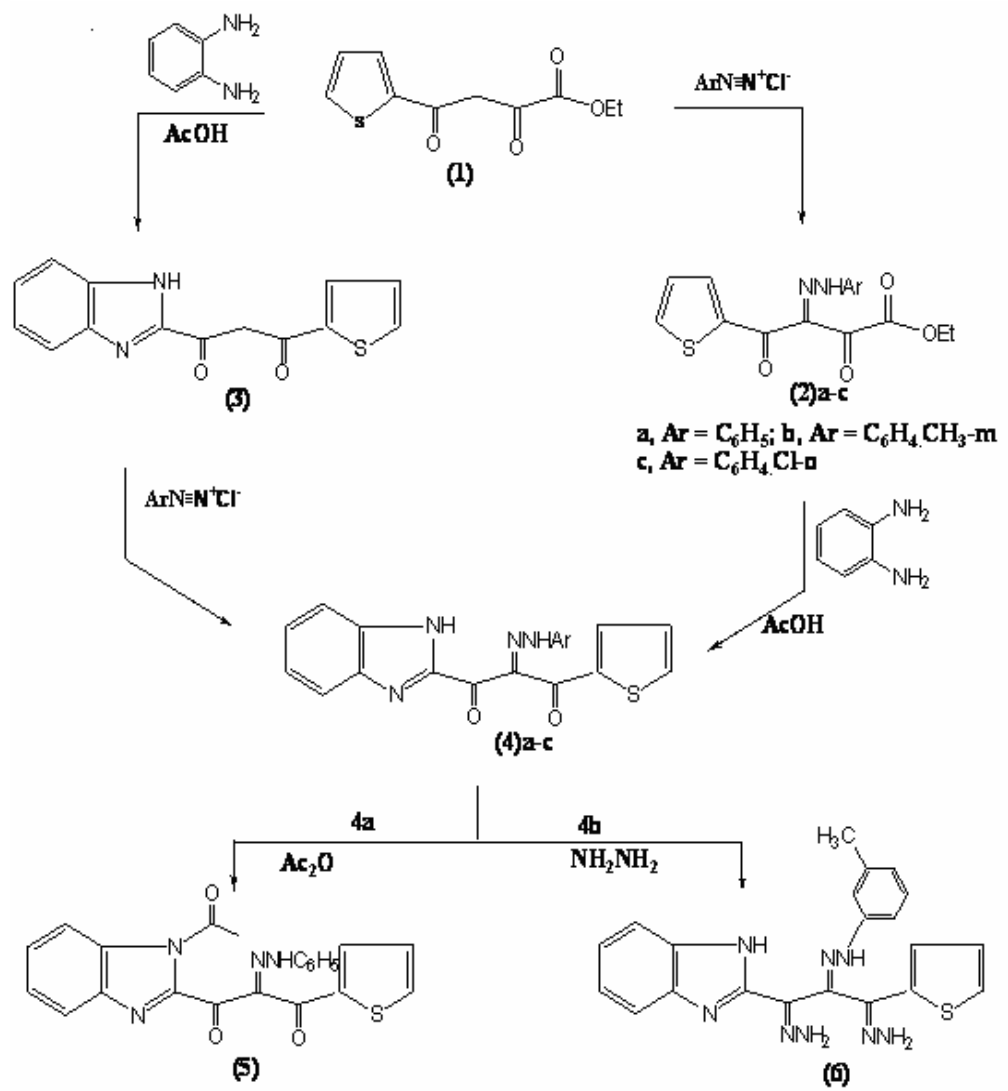

Scheme 1

2-Methyl-3-(2-thionyl)-4H-4-oxopyrrolo[1,2a]-benzimidazole (10) was prepared by the cyclocondensation of 2-[(2-thionyl)acetyl]benzimidazole (3) with acetic anhydride in the presence of fused sodium acetate under reflux. 2-[(2-thionyl)-acetyl]benzimidazole (3) reacted with phosphorus oxychlorid under reflux on a water bath affording 2-(2-thionyl)-4H-4-oxopyrrolo[1,2-a]benzimidazole (11) (Scheme 2). 


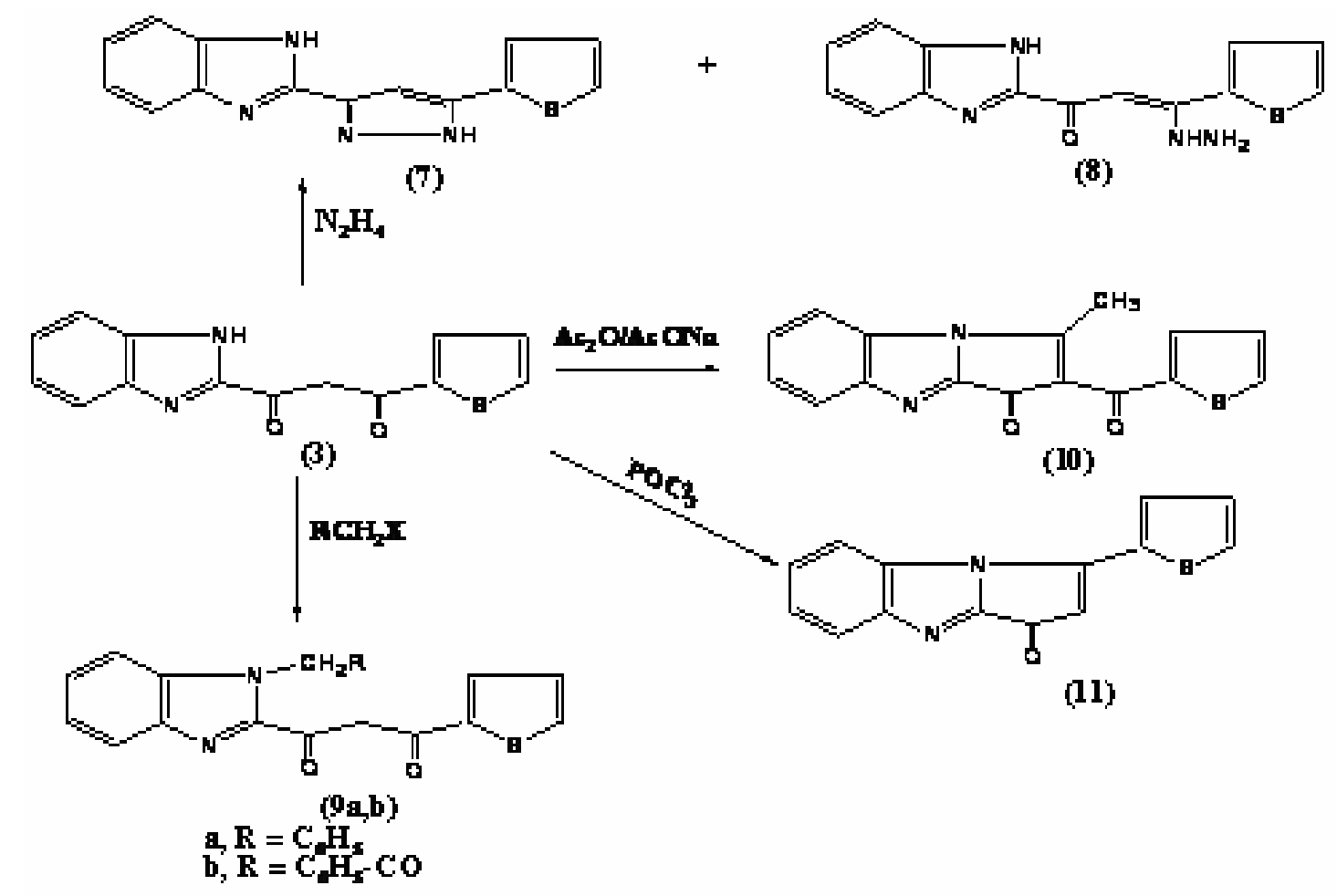

Scheme 2

\section{Mass spectroscopy}

The mass spectral decomposition modes of various benzimidazaole derivatives containing thiophene substituents have been suggested and investigated.

\section{Compounds 2, 4 and 5}

Table 1 lists the $m / z$ (relative abundance, \%) values of the principal fragments of the synthesized compounds $\mathbf{2 ,} \mathbf{4}$ and $\mathbf{5}$.

The mass spectra of compounds $\mathbf{2}, \mathbf{4}$ and $\mathbf{5}$ show relatively strong molecular ions and peaks typical of a cleavage and rearrangement processes type fragmentation. Thus, compounds $2 \mathrm{a}-\mathrm{c}$ showed an intense molecular ion peak at 330, 344 and 364 corresponding to the molecular formulas $\mathrm{C}_{16} \mathrm{H}_{14} \mathrm{~N}_{2} \mathrm{O}_{4} \mathrm{~S}$, $\mathrm{C}_{17} \mathrm{H}_{16} \mathrm{~N}_{2} \mathrm{O}_{4} \mathrm{~S}$ and $\mathrm{C}_{16} \mathrm{H}_{13} \mathrm{~N}_{2} \mathrm{ClO}_{4} \mathrm{~S}$, respectively. From a study of the mass spectra of compounds 2a-c, it was found that the molecular ion for all these compounds fragmented further and involved two various possible pathways as illustrated by Scheme 3 showing representative examples.

However, the molecule of $\mathrm{m} / \mathrm{z} 344$ (Fig.1) fragmented via the pathway A to give the ion of $\mathrm{m} / \mathrm{z}$ 298 , which fragmented further to give ion of $\mathrm{m} / \mathrm{z}$ 270 by losing CO. The ion of $\mathrm{m} / z 270$ was broken to give the ion $\mathrm{m} / \mathrm{z} 242$ by losing CO. The ion of $m / z 242$ underwent loss of $\mathrm{C}_{8} \mathrm{H}_{7} \mathrm{~N}_{2}$ to give a stable peak at $m / z 111$, which fragmented further to give a peak at $m / z 83$ by losing CO.

Subsequently, the molecular ion $\mathrm{m} / \mathrm{z} 344$ fragmented via the pathway $B$ to give a fragmented ion of $\mathrm{m} / \mathrm{z} 300$ by losing ethylene epoxide. Ion $\mathrm{m} / \mathrm{z}$ 300 fragmented to give an ion of $m / z 271$, which lost CO to give a fragmented of $m / z 243$. This fragmented led to $m / z 106,91$ and $m / z 65$, respectively.

The mass spectra of compounds $\mathbf{4 a}, \mathbf{4 b}$ and 4c showed intense molecular ion peaks at $\mathrm{m} / \mathrm{z} 374$, 388 and 408, consistent with the molecular formulas $\mathrm{C}_{20} \mathrm{H}_{14} \mathrm{~N}_{4} \mathrm{O}_{2} \mathrm{~S}, \mathrm{C}_{21} \mathrm{H}_{16} \mathrm{~N}_{4} \mathrm{O}_{2} \mathrm{~S}$ and $\mathrm{C}_{20} \mathrm{H}_{13} \mathrm{~N}_{4} \mathrm{ClO}_{2} \mathrm{~S}$, respectively.

The molecular ion of compound 4a (Fig. 2) fragmented further and involved two possible pathways as illustrated in Scheme 4 as representative example.

The molecular ion of $m / z 374$ fragmented via the pathway A to give stable ion of $m / z 111$ by losing 1-phenylamino-4-oxopyrazolo[1,2-a]benzimidazole radical. The stable ion of $m / z 111$ was broken to give the $m / z 83$ by losing $\mathrm{CO}$ group.

Accordingly, the same ion of $\mathrm{m} / \mathrm{z} 374$ fragmented via the pathway B by losing thionyl radical to give an ion $\mathrm{m} / \mathrm{z} 263$, which lost carbon monoxide to give an ion of $m / z 235$. This fragmentation led to ion of $\mathrm{m} / \mathrm{z} 119,92$ and $\mathrm{m} / \mathrm{z} 77$, respectively. 
Table 1

EI Spectra (70 eV) of compounds 2, 4 and 5

\begin{tabular}{|c|c|c|c|c|c|c|}
\hline \multirow[t]{2}{*}{ No. } & \multirow[t]{2}{*}{$\mathbf{M}^{+}$} & \multicolumn{2}{|c|}{ Pathway A } & \multicolumn{2}{|c|}{ Pathway B } & \multirow[t]{2}{*}{ Other ions } \\
\hline & & M- & $\mathrm{m} / \mathrm{z}$ & M- & $\mathrm{m} / \mathrm{z}$ & \\
\hline \multirow[t]{10}{*}{$2 \mathrm{a}$} & {$\left[\mathrm{C}_{16} \mathrm{H}_{14} \mathrm{~N}_{2} \mathrm{O}_{4} \mathrm{~S}\right]^{+}$} & $\mathrm{C}_{2} \mathrm{H}_{5} \mathrm{OH}$ & {$\left[\mathrm{C}_{14} \mathrm{H}_{8} \mathrm{~N}_{2} \mathrm{O}_{3} \mathrm{~S}\right]^{+}$} & $\mathrm{C}_{2} \mathrm{H}_{4} \mathrm{O}$ & {$\left[\mathrm{C}_{14} \mathrm{H}_{10} \mathrm{~N}_{2} \mathrm{O}_{3} \mathrm{~S}\right]^{+}$} & $331\left(\mathrm{M}^{+}+1,5.2\right), 296(1.2), 287(3$ \\
\hline & $330(15.00)$ & & $284(7.10)$ & & $286(6.30)$ & 2), 285(4.1), 283(2.1), 258(2.2), \\
\hline & & $\mathrm{CO}$ & {$\left[\mathrm{C}_{13} \mathrm{H}_{8} \mathrm{~N}_{2} \mathrm{O}_{2} \mathrm{~S}\right]^{++}$} & $\mathrm{CHO}$ & {$\left[\mathrm{C}_{13} \mathrm{H}_{9} \mathrm{~N}_{2} \mathrm{O}_{2} \mathrm{~S}\right]^{+}$} & 255(3.9), 227(1.6), 226(2.1) \\
\hline & & & $256(15.30)$ & & $257(8.35)$ & $213(1.6), 212(2.2), 117(1.3)$ \\
\hline & & $\mathrm{CO}$ & {$\left[\mathrm{C}_{12} \mathrm{H}_{8} \mathrm{~N}_{2} \mathrm{OS}\right]^{+}$} & $\mathrm{CO}$ & {$\left[\mathrm{C}_{12} \mathrm{H}_{9} \mathrm{~N}_{2} \mathrm{OS}\right]^{+}$} & $116(2.1), 112(17.2), 110(22.1)$ \\
\hline & & & $228(8.30)$ & & $229(6.35)$ & $91(11.2), 90(8.9), 84(2.2)$ \\
\hline & & $\mathrm{C}_{7} \mathrm{H}_{5} \mathrm{~N}_{2}$ & {$\left[\mathrm{C}_{5} \mathrm{H}_{3} \mathrm{OS}\right]^{+}$} & $\mathrm{C}_{6} \mathrm{H}_{3} \mathrm{NOS}$ & {$\left[\mathrm{C}_{6} \mathrm{H}_{6} \mathrm{~N}\right]^{+}$} & $82(6.3), 77(22), 76(11.3)$ \\
\hline & & & $111(100)$ & & $92(22.30)$ & $75(2.5), 65(11.2), 64(9.2)$, \\
\hline & & $\mathrm{CO}$ & {$\left[\mathrm{C}_{4} \mathrm{H}_{3} \mathrm{~S}\right]^{+}$} & $\mathrm{NH}$ & {$\left[\mathrm{C}_{6} \mathrm{H}_{5}\right]^{+}$} & $51(12.7)$ \\
\hline & & & $83(19.10)$ & & $77(36.70)$ & \\
\hline \multirow[t]{10}{*}{$2 b$} & {$\left[\mathrm{C}_{17} \mathrm{H}_{16} \mathrm{~N}_{2} \mathrm{O}_{4} \mathrm{~S}\right]^{++}$} & $\mathrm{C}_{2} \mathrm{H}_{5} \mathrm{OH}$ & {$\left[\mathrm{C}_{15} \mathrm{H}_{10} \mathrm{~N}_{2} \mathrm{O}_{3} \mathrm{~S}\right]^{+}$} & $\mathrm{C}_{2} \mathrm{H}_{4} \mathrm{O}$ & {$\left[\mathrm{C}_{15} \mathrm{H}_{12} \mathrm{~N}_{2} \mathrm{O}_{3} \mathrm{~S}\right]^{+}$} & $330(4.3), 299(2.3), 297(4.7)$ \\
\hline & $344(9.30)$ & & $298(5.10)$ & & $300(4.00)$ & $244(2.8), 189(1.4), 188(2.8)$, \\
\hline & & $\mathrm{CO}$ & {$\left[\mathrm{C}_{14} \mathrm{H}_{10} \mathrm{~N}_{2} \mathrm{O}_{2} \mathrm{~S}\right]^{+}$} & $\mathrm{CHO}$ & {$\left[\mathrm{C}_{14} \mathrm{H}_{11} \mathrm{~N}_{2} \mathrm{O}_{2} \mathrm{~S}\right]^{+}$} & 159(2. 0$), 153(1.5), 133(5.0)$, \\
\hline & & & $270(2.40)$ & & $271(4.01)$ & $132(3.4), 119(3.9), 112(7.5)$ \\
\hline & & $\mathrm{CO}$ & {$\left[\mathrm{C}_{13} \mathrm{H}_{10} \mathrm{~N}_{2} \mathrm{OS}\right]^{+}$} & $\mathrm{CO}$ & {$\left[\mathrm{C}_{13} \mathrm{H}_{11} \mathrm{~N}_{2} \mathrm{OS}\right]^{+}$} & $110(3.1), 107(2.5), 104(6.6)$ \\
\hline & & & $242(12.60)$ & & $243(4.70)$ & $90(2.2), 82(2.0), 77(16.8)$ \\
\hline & & $\mathrm{C}_{8} \mathrm{H}_{7} \mathrm{~N}_{2}$ & {$\left[\mathrm{C}_{5} \mathrm{H}_{3} \mathrm{OS}\right]^{+}$} & $\mathrm{C}_{6} \mathrm{H}_{3} \mathrm{NOS}$ & {$\left[\mathrm{C}_{7} \mathrm{H}_{8} \mathrm{~N}\right]^{+}$} & $65(11.5), 64(3.1), 52(6$. \\
\hline & & & $111(100)$ & & $106(9.80)$ & $1), 51(8.6)$ \\
\hline & & $\mathrm{CO}$ & {$\left[\mathrm{C}_{4} \mathrm{H}_{3} \mathrm{~S}\right]^{+}$} & $\mathrm{NH}$ & {$\left[\mathrm{C}_{7} \mathrm{H}_{7}\right]^{+}$} & \\
\hline & & & $83(9.10)$ & & $91(15.50)$ & \\
\hline \multirow[t]{13}{*}{$2 \mathrm{c}$} & {$\left[\mathrm{C}_{16} \mathrm{H}_{13} \mathrm{~N}_{2} \mathrm{ClO}_{4} \mathrm{~S}^{+}\right.$} & $\mathrm{C}_{2} \mathrm{H}_{5} \mathrm{OH}$ & {$\left[\mathrm{C}_{14} \mathrm{H}_{7} \mathrm{~N}_{+} \mathrm{ClO}_{3} \mathrm{~S}\right]$} & $\mathrm{C}_{2} \mathrm{H}_{4} \mathrm{O}$ & {$\left[\mathrm{C}_{14} \mathrm{H}_{9} \mathrm{~N}_{2} \mathrm{ClO}_{3} \mathrm{~S}\right.$} & $366\left(\mathrm{M}^{+}+2,5.2\right), 350(5.5)$ \\
\hline & & $\mathrm{CO}$ & $318(9.30)$ & $\mathrm{CHO}$ & $\begin{array}{c}]^{+} \\
320(5.30)\end{array}$ & $\begin{array}{c}349(4.8), 292(2.9), 289(4.7), \\
279(2.7), 264(2.4), 261(5.8)\end{array}$ \\
\hline & & & {$\left[\mathrm{C}_{13} \mathrm{H}_{7} \mathrm{~N}_{2} \mathrm{ClO}_{2} \mathrm{~S}\right]$} & & {$\left[\mathrm{C}_{13} \mathrm{H}_{8} \mathrm{~N}_{2} \mathrm{ClO}_{2} \mathrm{~S}\right.$} & $209(2.5), 208(2.7), 153$ \\
\hline & & $\mathrm{CO}$ & & $\mathrm{CO}$ & & $(5.3), 152(2.5), 140(1.5), 139$ \\
\hline & & & $290(7.30)$ & & $291(6.30)$ & $(5.8), 125(3.4), 112(7.3)$ \\
\hline & & $\mathrm{C}_{7} \mathrm{H}_{4} \mathrm{ClN}_{2}$ & $\begin{array}{c}{\left[\mathrm{C}_{12} \mathrm{H}_{7} \mathrm{~N}_{2} \mathrm{ClOS}\right]^{+}} \\
262(7.40)\end{array}$ & $\mathrm{C}_{6} \mathrm{H}_{3} \mathrm{NOS}$ & {$\left[\mathrm{C}_{12} \mathrm{H}_{8} \mathrm{~N}_{+} \mathrm{ClOS}\right]$} & $\begin{array}{c}110(1.8), 99(10.2), 91(2.9) \\
90(7.3), 84(2.8)\end{array}$ \\
\hline & & $\mathrm{CO}$ & {$\left[\mathrm{C}_{5} \mathrm{H}_{3} \mathrm{OS}\right]^{+}$} & $\mathrm{NH}$ & $263(3.00)$ & 76(1.9), 65(2.2), 64(5.7), \\
\hline & & & $111(100)$ & & {$\left[\mathrm{C}_{6} \mathrm{H}_{5} \mathrm{NCl}\right]^{+}$} & $63(8.9), 54(6.1), 51(12.7)$ \\
\hline & & & {$\left[\mathrm{C}_{4} \mathrm{H}_{3} \mathrm{~S}\right]^{+}$} & $\mathrm{HCl}$ & $92(22.30)$ & \\
\hline & & & $83(19.10)$ & & {$\left[\mathrm{C}_{6} \mathrm{H}_{4} \mathrm{Cl}\right]^{+}$} & \\
\hline & & & & & $111(100)$ & \\
\hline & & & & & {$\left[\mathrm{C}_{6} \mathrm{H}_{3}\right]^{+}$} & \\
\hline & & & & & $75(7.00)$ & \\
\hline \multirow[t]{10}{*}{$4 \mathrm{a}$} & {$\left[\mathrm{C}_{20} \mathrm{H}_{14} \mathrm{~N}_{4} \mathrm{O}_{2} \mathrm{~S}\right]^{+}$} & $\mathrm{C}_{15} \mathrm{H}_{11} \mathrm{~N}_{4} \mathrm{O}$ & {$\left[\mathrm{C}_{5} \mathrm{H}_{3} \mathrm{OS}\right]^{+}$} & $\mathrm{C}_{5} \mathrm{H}_{3} \mathrm{OS}$ & {$\left[\mathrm{C}_{15} \mathrm{H}_{11} \mathrm{~N}_{4} \mathrm{O}\right]^{+}$} & $375\left(\mathrm{M}^{+}+1,25.3\right), 373(13.7), 357$ \\
\hline & $374(72.20)$ & & $111(100)$ & & $263(4.40)$ & (13.8), 356(7.0), 342(3.2) \\
\hline & & $\mathrm{CO}$ & {$\left[\mathrm{C}_{4} \mathrm{H}_{3} \mathrm{~S}\right]^{+}$} & $\mathrm{CO}$ & {$\left[\mathrm{C}_{14} \mathrm{H}_{11} \mathrm{~N}_{4}\right]^{+}$} & 341(14. 2), 269(2.7), 254(3.5), \\
\hline & & & $83(19.10)$ & & $235(2.7)$ & $242(2.6), 241(9.5), 234(1.7)$ \\
\hline & & & & $\mathrm{C}_{7} \mathrm{H}_{4} \mathrm{~N}_{2}$ & {$\left[\mathrm{C}_{7} \mathrm{H}_{7} \mathrm{~N}_{2}\right]^{+}$} & 213(5.0), $208(3.5), 206(3.0)$ \\
\hline & & & & & $119(1.4)$ & $197(2.2), 176(7.8), 175(53.4)$ \\
\hline & & & & $\mathrm{HCN}$ & {$\left[\mathrm{C}_{6} \mathrm{H}_{6} \mathrm{~N}\right]^{+}$} & 174(5.7), 172(23.4), 158(2.0) \\
\hline & & & & & $92(27.70)$ & 157(1.5), 144(2.8), $143(2.5)$, \\
\hline & & & & $\mathrm{NH}$ & {$\left[\mathrm{C}_{6} \mathrm{H}_{5}\right]^{+}$} & $117(2.5), 116(1.5), 91(8.0)$, \\
\hline & & & & & $77(33.20)$ & 84(3.2), 76(4.0), 65(38.1), \\
\hline \multirow[t]{12}{*}{$4 \mathrm{~b}$} & {$\left[\mathrm{C}_{21} \mathrm{H}_{16} \mathrm{~N}_{4} \mathrm{O}_{2} \mathrm{~S}^{++}\right.$} & $\mathrm{C}_{16} \mathrm{H}_{13} \mathrm{~N}_{4} \mathrm{O}$ & {$\left[\mathrm{C}_{5} \mathrm{H}_{3} \mathrm{OS}\right]^{+}$} & $\mathrm{C}_{5} \mathrm{H}_{3} \mathrm{OS}$ & {$\left[\mathrm{C}_{16} \mathrm{H}_{13} \mathrm{~N}_{4} \mathrm{O}\right]^{+}$} & $389\left(\mathrm{M}^{+}+1,12.6\right), 371(10.2)$ \\
\hline & $388(48.00)$ & & $111(100)$ & & $277(1.40)$ & $370(5.2), 355(13.5), 297(3.2)$ \\
\hline & & $\mathrm{CO}$ & {$\left[\mathrm{C}_{4} \mathrm{H}_{3} \mathrm{~S}\right]^{+}$} & $\mathrm{CO}$ & {$\left[\mathrm{C}_{15} \mathrm{H}_{13} \mathrm{~N}_{4}\right]^{+}$} & 276(1.8), 248(2.0), 241(9.9) \\
\hline & & & $83(19.60)$ & & $249(4.2)$ & $240(5.3), 234(3.0), 223(2.2)$ \\
\hline & & & & $\mathrm{C}_{7} \mathrm{H}_{4} \mathrm{~N}_{2}$ & {$\left[\mathrm{C}_{8} \mathrm{H}_{9} \mathrm{~N}_{2}\right]^{+}$} & 213(4.4), $212(3.4), 206(3.2)$, \\
\hline & & & & & $133(1.2)$ & 205(3.1), 197(2.4), 190(6.3), \\
\hline & & & & $\mathrm{HCN}$ & {$\left[\mathrm{C}_{7} \mathrm{H}_{8} \mathrm{~N}\right]^{+}$} & 189(50.8), 188(6.4), 174(4.5) \\
\hline & & & & & $106(17.70)$ & 173(4.8), 172(21.2), $156(2.0)$ \\
\hline & & & & $\mathrm{NH}$ & {$\left[\mathrm{C}_{7} \mathrm{H}_{7}\right]^{+}$} & $145(3.1), 144(3.8), 143(4.1)$ \\
\hline & & & & & $91(26.80)$ & $129(2.3), 118(2.7), 117(3.2)$ \\
\hline & & & & $\mathrm{C}_{2} \mathrm{H}_{2}$ & {$\left[\mathrm{C}_{5} \mathrm{H}_{5}\right]^{+}$} & 116(3.0), 107(4.6), 90(13.6), \\
\hline & & & & & $65(20.00)$ & $84(3.1), 66(13.3), 51(10.3)$ \\
\hline
\end{tabular}




\begin{tabular}{|c|c|c|c|c|c|c|}
\hline $4 c$ & $\begin{array}{c}{\left[\mathrm{C}_{20} \mathrm{H}_{13} \mathrm{~N}_{4} \mathrm{ClO}_{2} \mathrm{~S}^{+}\right]^{+}} \\
408.5(11.50)\end{array}$ & $\begin{array}{c}\mathrm{C}_{15} \mathrm{H}_{10} \mathrm{~N}_{4} \mathrm{ClO} \\
\mathrm{CO}\end{array}$ & $\begin{array}{c}{\left[\mathrm{C}_{5} \mathrm{H}_{3} \mathrm{OS}\right]^{+}} \\
111(100) \\
{\left[\mathrm{C}_{4} \mathrm{H}_{3} \mathrm{~S}\right]^{+}} \\
83(16.10)\end{array}$ & $\begin{array}{c}\mathrm{C}_{5} \mathrm{H}_{3} \mathrm{OS} \\
\mathrm{CO} \\
\mathrm{C}_{7} \mathrm{H}_{4} \mathrm{~N}_{2} \\
\mathrm{HCN} \\
\mathrm{NH} \\
\mathrm{HCl}\end{array}$ & $\begin{array}{c}{\left[\mathrm{C}_{15} \mathrm{H}_{10} \mathrm{~N}_{4} \mathrm{ClO}\right]^{+}} \\
297(1.30) \\
{\left[\mathrm{C}_{14} \mathrm{H}_{10} \mathrm{~N}_{4} \mathrm{Cl}\right]^{+}} \\
269(3.1) \\
{\left[\mathrm{C}_{7} \mathrm{H}_{6} \mathrm{~N}_{2} \mathrm{Cl}\right]^{+}} \\
153(1.2) \\
{\left[\mathrm{C}_{6} \mathrm{H}_{5} \mathrm{NCl}\right]^{+}} \\
126(12.20) \\
{\left[\mathrm{C}_{6} \mathrm{H}_{4} \mathrm{Cl}\right]^{+}} \\
111(100) \\
{\left[\mathrm{C}_{6} \mathrm{H}_{3}\right]^{+}} \\
75(9.40)\end{array}$ & $\begin{array}{c}410\left(\mathrm{M}^{+}+2,41.5\right), 407(11.7), 390 \\
(3.3), 375(3.8), 374(2.9), 373(2 . \\
0), 268(2.0), 254(2.8), 253(3.5), \\
251(3.4), 250(2.9), 241(7.6), \\
240(6.6), 213(3.5), 212(3.0), \\
211(6.6), 210(8.0), 209(17.5), \\
208(18.2), 187(1.6), 186(5.6), \\
185(4.9), 173(7.8), 172(17.5), \\
171(20.8), 158(1.4), 157(3.5), \\
144(4.8), 125(16.0), 118(3.5), \\
113(10.4), 110(84.4), 98(16.5), \\
82(12.6), 90(16.9)\end{array}$ \\
\hline 5 & $\begin{array}{c}{\left[\mathrm{C}_{22} \mathrm{H}_{16} \mathrm{~N}_{4} \mathrm{O}_{3} \mathrm{~S}\right]^{+}} \\
416(23.00)\end{array}$ & $\begin{array}{c}\mathrm{C}_{5} \mathrm{H}_{3} \mathrm{OS} \\
\mathrm{C}_{6} \mathrm{H}_{6} \mathrm{~N} \\
\mathrm{CH}_{2} \mathrm{CO} \\
\mathrm{HCN} \\
\mathrm{CN} \\
\mathrm{CO}\end{array}$ & $\begin{array}{c}{\left[\mathrm{C}_{17} \mathrm{H}_{13} \mathrm{~N}_{4} \mathrm{O}_{2}\right]^{+}} \\
305(100) \\
{\left[\mathrm{C}_{11} \mathrm{H}_{7} \mathrm{~N}_{3} \mathrm{O}_{2}\right]^{+}} \\
213(8.80) \\
{\left[\mathrm{C}_{9} \mathrm{H}_{5} \mathrm{~N}_{3} \mathrm{O}\right]^{+}} \\
171(5.1) \\
{\left[\mathrm{C}_{8} \mathrm{H}_{4} \mathrm{~N}_{2} \mathrm{O}\right]^{+}} \\
144(13.1) \\
{\left[\mathrm{C}_{7} \mathrm{H}_{4} \mathrm{NO}\right]^{+}} \\
118(5.1) \\
{\left[\mathrm{C}_{6} \mathrm{H}_{4} \mathrm{~N}\right]^{+}} \\
90(26.7) \\
\end{array}$ & $\begin{array}{c}\mathrm{C}_{17} \mathrm{H}_{13} \mathrm{~N}_{4} \mathrm{O}_{2} \\
\mathrm{CO}\end{array}$ & $\begin{array}{c}{\left[\mathrm{C}_{5} \mathrm{H}_{3} \mathrm{OS}\right]^{+}} \\
111(20.90) \\
{\left[\mathrm{C}_{4} \mathrm{H}_{3} \mathrm{~S}\right]^{+}} \\
83(9.90)\end{array}$ & $\begin{array}{c}417\left(\mathrm{M}^{+}+1,13.1\right), 374(44.1), 373 \\
(34.2), 358(3.7), 57(70.1), 347 \\
(15.8), 346(11.0), 306(40.4), \\
273(5.6), 254(8.8), 243(6.1), \\
241(12.8), 214(4.0), 74(11.2), \\
173(12.3), 72(31.6), 159(5.3), \\
155(5.9), 143(4.3), 135(8.6), \\
134(6.4), 131(6.7), 130(8.8), \\
128(6.7), 119(4.0), 117(4.8), \\
110(8.0), 105(5.3), 92(26.7), \\
91(13.4), 84(2.1), 77(17.9), \\
65(93.0), 63(2.9), 52(7.5) .\end{array}$ \\
\hline
\end{tabular}

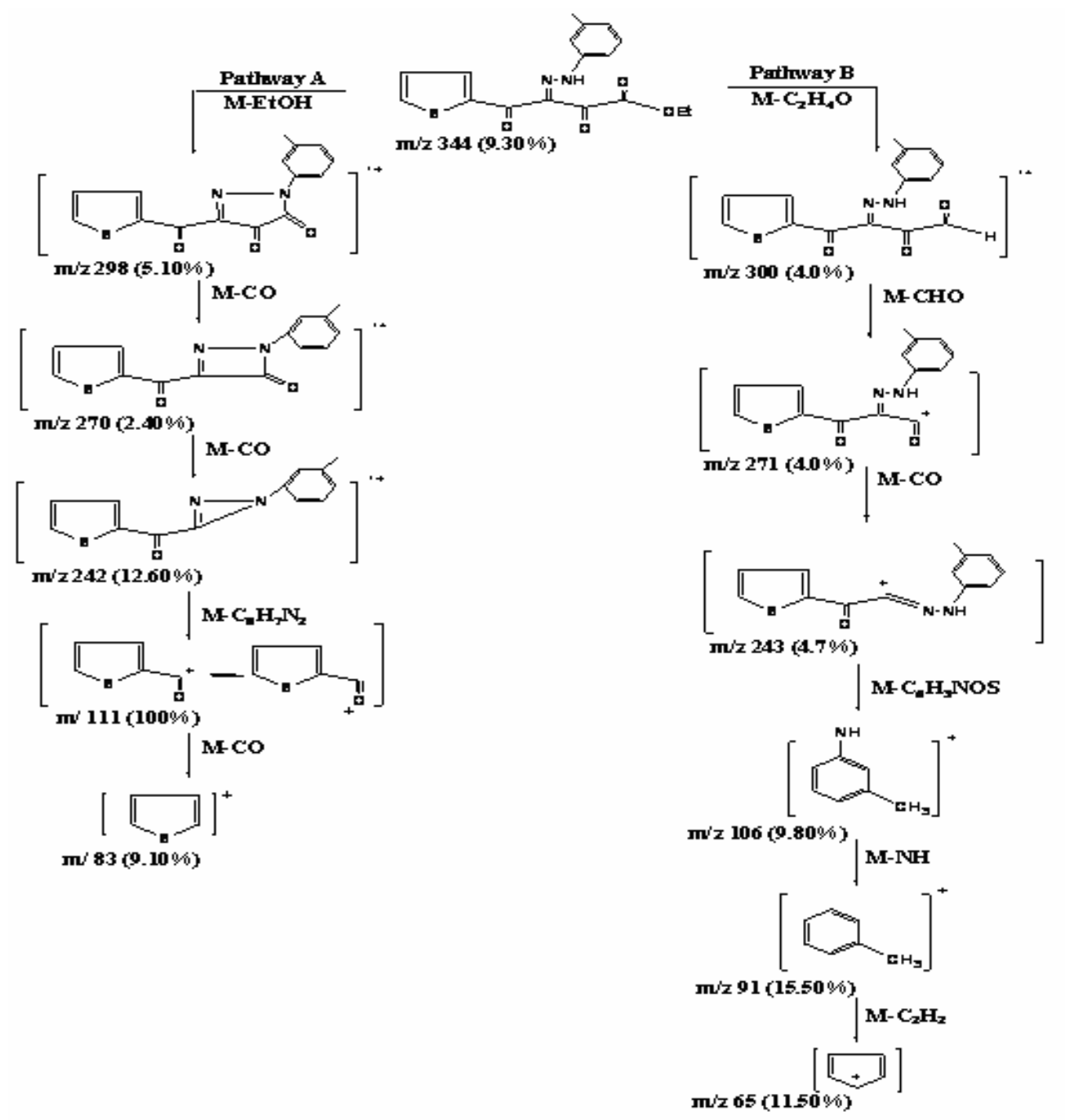

Scheme 3. Mass fragmentation pattern of compound $2 \mathrm{~b}$ 


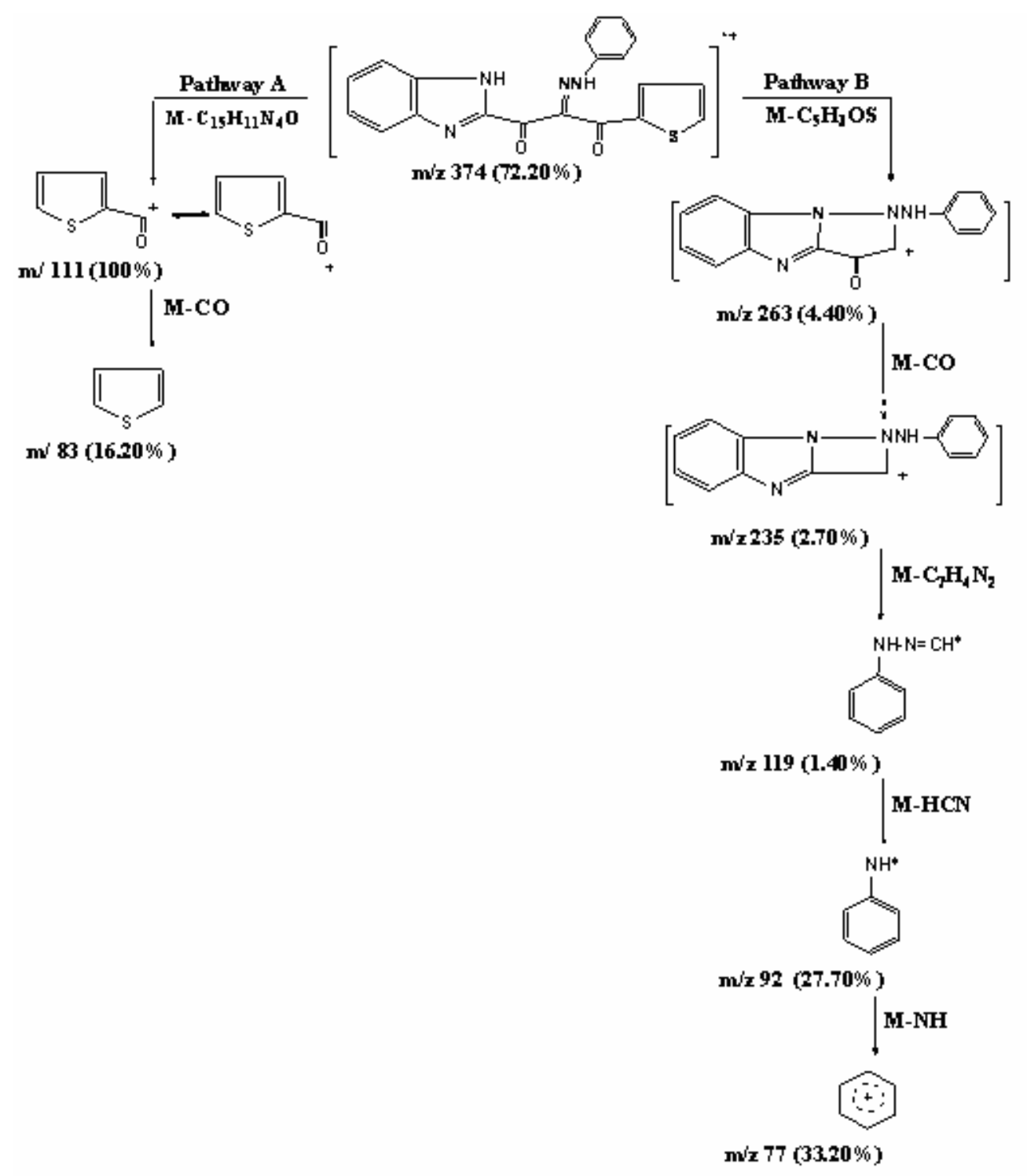

Scheme 4. Mass fragmentation pattern of compound 4a

The molecular ion peak of compound $\mathbf{5}$ was observed at $m / z 416$ (Fig. 3), corresponding to the molecular formula $\mathrm{C}_{22} \mathrm{H}_{16} \mathrm{~N}_{4} \mathrm{O}_{3} \mathrm{~S}$. The ion peak of compound 5 at $\mathrm{m} / \mathrm{z} 416$ fragmented further and involved two possible pathways as illustrated in Scheme 5. The ion of $m / z 416$ fragmented via the pathway A to give a stable fragmented ion of $\mathrm{m} / \mathrm{z}$ 305 by losing 2-thionyl radical cation, the ion of $\mathrm{m} / \mathrm{z} 305$ fragmented to give an ion of $\mathrm{m} / \mathrm{z} 213$, which lost $\mathrm{CH}_{2} \mathrm{CO}$ to give a fragmented ion of $\mathrm{m} / \mathrm{z}$ 171. This fragmentation led to $\mathrm{m} / z 144,118,90$ and $m / z 64$, respectively.

Subsequently, the molecular ion $\mathrm{m} / z 416$ fragmented via the pathway $\mathrm{B}$ to give the ion of $\mathrm{m} / \mathrm{z} 111$, which fragmented further to give ion of $\mathrm{m} / \mathrm{z} 83$ by losing CO.

\section{Compounds 3, 9a, 9b, 10 and 11}

The mass spectra of the synthesized compounds 3, 9a, 9b, 10 and 11 showed intense molecular ion peaks at $\mathrm{m} / \mathrm{z} 270,360,388,294$ and $\mathrm{m} / \mathrm{z}$ 252, consistent with the molecular formulas $\mathrm{C}_{14} \mathrm{H}_{10} \mathrm{~N}_{2} \mathrm{O}_{2} \mathrm{~S}, \quad \mathrm{C}_{21} \mathrm{H}_{16} \mathrm{~N}_{2} \mathrm{O}_{2} \mathrm{~S}, \quad \mathrm{C}_{22} \mathrm{H}_{16} \mathrm{~N}_{2} \mathrm{O}_{3} \mathrm{~S}$, $\mathrm{C}_{16} \mathrm{H}_{10} \mathrm{~N}_{2} \mathrm{O}_{2} \mathrm{~S}$ and $\mathrm{C}_{14} \mathrm{H}_{8} \mathrm{~N}_{2} \mathrm{OS}$, respectively.

Table 2 list the $\mathrm{m} / \mathrm{z}$ (relative abundance, \%) values of the principal fragments of the prepared compounds 3, 9, 10 and 11 that show relatively strong molecular ion and peaks typical of a cleavage and rearrangement process type fragmentation. 


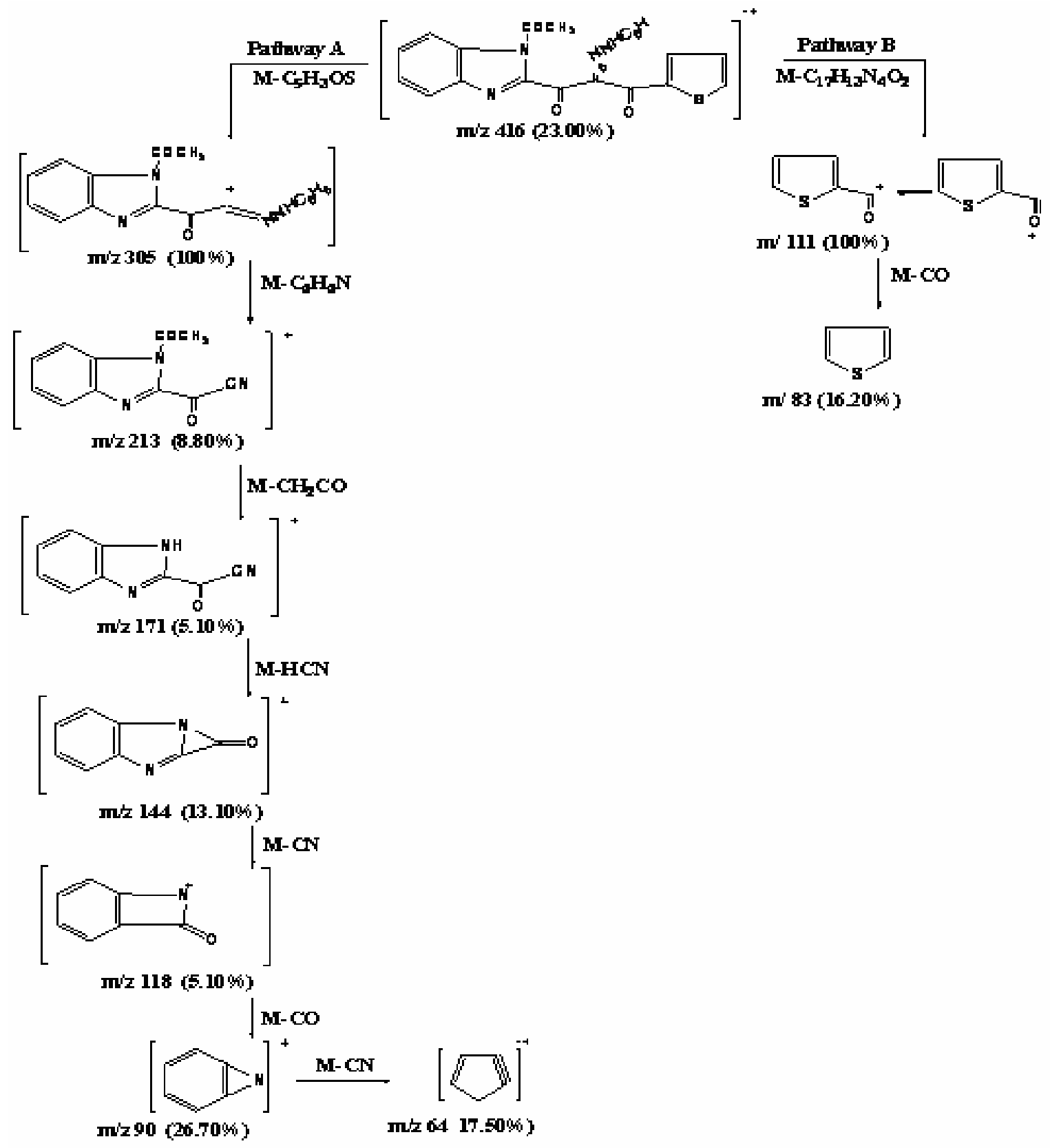

Scheme 5. Mass fragmentation pattern of compound 5

From a study of the mass spectra of compounds $3,9 \mathrm{a}$ and $9 \mathrm{~b}$, it was found that the molecular ion for all these compounds fragmented further and involved two various possible pathway as illustrated by scheme 6 ; however, the molecular ion of compound 3 of $m / z 270$ (Fig. 4) fragmented via the pathway A to give a stable ion of $m / z$ 111, which fragmented further to give ion of $m / z 83$ by losing CO. Accordingly, the same ion of $\mathrm{m} / \mathrm{z} 270$ was broken via pathway B to give an ion $\mathrm{m} / z 186$ by losing thiophene molecule. The ion at $\mathrm{m} / \mathrm{z} 186$ underwent fragmentation to produce peaks at $\mathrm{m} / \mathrm{z}$ 158,130 and 103 by losing two carbon monoxide molecule and hydrogen cyanide molecule.

From a study of the mass spectra of compounds $9 \mathbf{a}$ and $9 \mathbf{b}$, it was found that the molecular ion for these two compounds had fragmented to ion of $\mathrm{m} / \mathrm{z} 270$, corresponding to the molecular ion of compound 3 by losing $\mathrm{C}_{7} \mathrm{H}_{6}$ and $\mathrm{C}_{8} \mathrm{H}_{6} \mathrm{O}$ molecular (Figs. 5 and 6) the ion of $\mathrm{m} / \mathrm{z} 270$ which has further broken via similar pathway of compound 3 . 
Table 2

EI Spectra (70 eV) of compounds 3, 9, 10 and 11

\begin{tabular}{|c|c|c|c|c|c|c|}
\hline \multirow[t]{2}{*}{ No. } & \multirow[t]{2}{*}{$\overline{\mathrm{M}^{+}}$} & \multicolumn{2}{|c|}{ Pathway A } & \multicolumn{2}{|c|}{ Pathway B } & \multirow[t]{2}{*}{ Other ions } \\
\hline & & M- & $\mathrm{m} / \mathrm{z}$ & M- & $\mathrm{m} / \mathrm{z}$ & \\
\hline 3 & 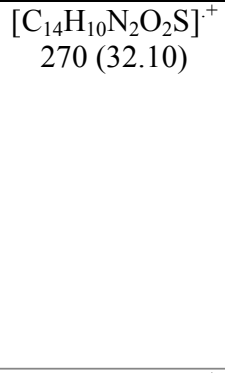 & $\begin{array}{r}\mathrm{C}_{4} \mathrm{H}_{4} \mathrm{~S} \\
\mathrm{CO} \\
\mathrm{CO} \\
\mathrm{HCN} \\
\mathrm{CN}\end{array}$ & 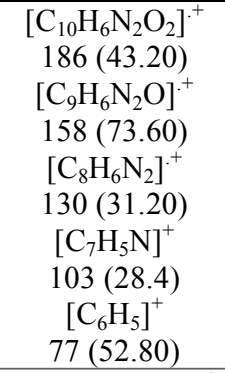 & $\begin{array}{c}\mathrm{C}_{9} \mathrm{H}_{7} \mathrm{~N}_{2} \mathrm{O} \\
\mathrm{CO}\end{array}$ & 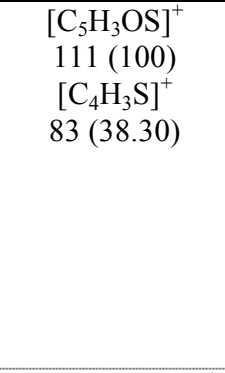 & $\begin{array}{c}271\left(\mathrm{M}^{+}+1,17.6\right), 241(18.5), \\
160(23.7), 131(11.1), \\
109(17.3), 108(12.3), \\
105(11.2), 104(28.4), \\
91(64.3), 90(33.3), 84(3.7), \\
79(22.2), 78(19.2), \\
76(18.5), 69(49.4), \\
64(32.1), 63(28.4), 57(45.7), \\
53(40.7), 51(19.8) .\end{array}$ \\
\hline $9 a$ & $\begin{array}{c}{\left[\mathrm{C}_{21} \mathrm{H}_{16} \mathrm{~N}_{2} \mathrm{O}_{2} \mathrm{~S}\right]^{+}} \\
360(9.70)\end{array}$ & $\begin{array}{c}\mathrm{C}_{7} \mathrm{H}_{6} \\
\mathrm{C}_{4} \mathrm{H}_{4} \mathrm{~S} \\
\mathrm{CO} \\
\mathrm{CO} \\
\mathrm{CN} \\
\mathrm{HCN}\end{array}$ & $\begin{array}{c}{\left[\mathrm{C}_{14} \mathrm{H}_{10} \mathrm{~N}_{2} \mathrm{O}_{2} \mathrm{~S}\right]^{++}} \\
270(100) \\
{\left[\mathrm{C}_{10} \mathrm{H}_{6} \mathrm{~N}_{2} \mathrm{O}_{2}\right]^{++}} \\
186(52.00) \\
{\left[\mathrm{C}_{9} \mathrm{H}_{6} \mathrm{~N}_{2} \mathrm{O}\right]^{+}} \\
158(26.70) \\
{\left[\mathrm{C}_{8} \mathrm{H}_{6} \mathrm{~N}_{2}\right]^{+}} \\
130(15.70) \\
{\left[\mathrm{C}_{7} \mathrm{H}_{6} \mathrm{~N}\right]^{+}} \\
104(8.00) \\
{\left[\mathrm{C}_{6} \mathrm{H}_{5}\right]^{+}} \\
77(18.60)\end{array}$ & $\begin{array}{c}\mathrm{C}_{7} \mathrm{H}_{6} \\
\mathrm{C}_{9} \mathrm{H}_{7} \mathrm{~N}_{2} \mathrm{O} \\
\mathrm{CO}\end{array}$ & $\begin{array}{c}{\left[\mathrm{C}_{14} \mathrm{H}_{10} \mathrm{~N}_{2} \mathrm{O}_{2} \mathrm{~S}\right]^{+}} \\
270(100) \\
{\left[\mathrm{C}_{5} \mathrm{H}_{3} \mathrm{OS}\right]^{+}} \\
111(100) \\
{\left[\mathrm{C}_{4} \mathrm{H}_{3} \mathrm{~S}\right]^{+}} \\
83(38.30)\end{array}$ & $\begin{array}{c}361\left(\mathrm{M}^{+}+1,4.7\right), 272(11.4), \\
271(23.3), 269(9.8), \\
241(10.7), 237(10.3), \\
213(3.8), 198(4.6), \\
187(13.2), 169(5.8), \\
159(14.2), 131(12.5), \\
129(3.7), 112(8.2), \\
103(12.8), 90(11.3), 84(7.3), \\
82(7.6), 78(6.4), 76(9.4), \\
69(4.9), 68(5.8), 64(13.1), \\
63(17.5), 58(13.1), \\
57(15.3), 53(13.5), 52 \\
(16.1), 51(12.4) .\end{array}$ \\
\hline $9 b$ & $\begin{array}{c}{\left[\mathrm{C}_{22} \mathrm{H}_{16} \mathrm{~N}_{2} \mathrm{O}_{3} \mathrm{~S}\right]^{+}} \\
388(32.80)\end{array}$ & $\begin{array}{c}\mathrm{C}_{8} \mathrm{H}_{6} \mathrm{O} \\
\mathrm{C}_{4} \mathrm{H}_{4} \mathrm{~S} \\
\mathrm{CO} \\
\mathrm{CO} \\
\mathrm{CN} \\
\mathrm{HCN}\end{array}$ & $\begin{array}{c}\left.\mathrm{C}_{14} \mathrm{H}_{10} \mathrm{~N}_{2} \mathrm{O}_{2} \mathrm{~S}\right]^{++} \\
270(61.50) \\
{\left[\mathrm{C}_{10} \mathrm{H}_{6} \mathrm{~N}_{2} \mathrm{O}_{2}\right]^{+}} \\
186(52.00) \\
{\left[\mathrm{C}_{9} \mathrm{H}_{6} \mathrm{~N}_{2} \mathrm{O}\right]^{+}} \\
158(26.70) \\
{\left[\mathrm{C}_{8} \mathrm{H}_{6} \mathrm{~N}_{2}\right]^{+}} \\
130(15.70) \\
{\left[\mathrm{C}_{7} \mathrm{H}_{6} \mathrm{~N}\right]^{+}} \\
104(8.00) \\
{\left[\mathrm{C}_{6} \mathrm{H}_{5}\right]^{+}} \\
77(18.60)\end{array}$ & $\begin{array}{c}\mathrm{C}_{8} \mathrm{H}_{6} \mathrm{O} \\
\mathrm{C}_{9} \mathrm{H}_{7} \mathrm{~N}_{2} \mathrm{O} \\
\mathrm{CO}\end{array}$ & $\begin{array}{c}{\left[\mathrm{C}_{14} \mathrm{H}_{10} \mathrm{~N}_{2} \mathrm{O}_{2} \mathrm{~S}\right]^{+}} \\
270(100) \\
{\left[\mathrm{C}_{5} \mathrm{H}_{3} \mathrm{OS}\right]^{+}} \\
111(100) \\
{\left[\mathrm{C}_{4} \mathrm{H}_{3} \mathrm{~S}\right]^{+}} \\
83(38.30)\end{array}$ & $\begin{array}{c}389\left(\mathrm{M}^{+}+1,18.9\right), 316(3.8), \\
360(5.7), 283(8.8), \\
271(10.6), 269(9.9), \\
242(10.3), 241(15.0), 187 \\
(10.7), 131(11.0), 129(3.9), \\
112(7.5), 105(53.1), \\
103(16.8), 91(5.4), 90(12.0), \\
84(5.8), 82(3.5), 78(8.2), \\
76(13.6), 65(10.1), \\
64(12.5), 63(16.1), 58(9.1), \\
53(10.1), 52(16.6), 51(41.9) .\end{array}$ \\
\hline 10 & $\begin{array}{c}{\left[\mathrm{C}_{16} \mathrm{H}_{10} \mathrm{~N}_{2} \mathrm{O}_{2} \mathrm{~S}\right]^{+}} \\
294(89.8)\end{array}$ & $\begin{array}{c}\mathrm{C}_{6} \mathrm{H}_{2} \\
\mathrm{CO} \\
\mathrm{HCN} \\
\mathrm{C}_{3} \mathrm{H}_{4} \mathrm{~N} \\
\mathrm{CO}\end{array}$ & $\begin{array}{c}{\left[\mathrm{C}_{10} \mathrm{H}_{8} \mathrm{~N}_{2} \mathrm{O}_{2} \mathrm{~S}\right]^{+}}_{220(100)} \\
{\left[\mathrm{C}_{9} \mathrm{H}_{8} \mathrm{~N}_{2} \mathrm{OS}\right]^{+}} \\
192(8.60) \\
{\left[\mathrm{C}_{8} \mathrm{H}_{7} \mathrm{NOS}\right]^{+}} \\
165(7.70) \\
{\left[\mathrm{C}_{5} \mathrm{H}_{3} \mathrm{OS}\right]^{+}} \\
111(61.2) \\
{\left[\mathrm{C}_{4} \mathrm{H}_{3} \mathrm{~S}\right]^{+}} \\
83(6.60)\end{array}$ & $\begin{array}{c}\mathrm{CH}_{3} \\
\mathrm{C}_{8} \mathrm{H}_{3} \mathrm{O}_{2} \mathrm{~S} \\
\mathrm{CN} \\
\mathrm{N}\end{array}$ & $\begin{array}{c}{\left[\mathrm{C}_{15} \mathrm{H}_{7} \mathrm{~N}_{2} \mathrm{O}_{2} \mathrm{~S}^{+}\right]^{+}} \\
279(73.70) \\
{\left[\mathrm{C}_{7} \mathrm{H}_{4} \mathrm{~N}_{2}\right]^{+}} \\
116(5.70) \\
{\left[\mathrm{C}_{6} \mathrm{H}_{4} \mathrm{~N}\right]^{+}} \\
90(38.80) \\
{\left[\mathrm{C}_{6} \mathrm{H}_{4}\right]^{+}} \\
76(7.20)\end{array}$ & $\begin{array}{c}295\left(\mathrm{M}^{+}+1,20.4\right), 243(57.8), \\
280(16.1), 271(2.3), \\
266(9.8), 265(7.2), \\
251(9.1), 237(10.9), 221 \\
(15.6), 219(10.4), 191(22.4), \\
190(3.8), 163(2.7), 164(5.4), \\
159(12.9), 147(7.0), \\
135(6.6), 133(8.8), 112(3.9), \\
104(10.0), 84(12.0), 82(3.0), \\
77(16.50), 64(15.0), \\
63(28.1), 52(7.0), 51(16.5)\end{array}$ \\
\hline 11 & $\begin{array}{c}{\left[\mathrm{C}_{14} \mathrm{H}_{8} \mathrm{~N}_{2} \mathrm{OS}\right]^{+}} \\
252(77.4)\end{array}$ & $\begin{array}{c}\mathrm{CN} \\
\mathrm{N} \\
\mathrm{C}_{2} \mathrm{H}_{2}\end{array}$ & $\begin{array}{c}{\left[\mathrm{C}_{13} \mathrm{H}_{8} \mathrm{~N}_{2} \mathrm{~S}\right]^{+}} \\
224(29.00) \\
{\left[\mathrm{C}_{7} \mathrm{H}_{4} \mathrm{~N}_{2}\right]^{+}} \\
116(7.40) \\
{\left[\mathrm{C}_{6} \mathrm{H}_{4} \mathrm{~N}\right]^{+}} \\
90(32.00) \\
{\left[\mathrm{C}_{6} \mathrm{H}_{4}\right]^{+}} \\
76(14.10) \\
{\left[\mathrm{C}_{4} \mathrm{H}_{2}\right]^{+}} \\
50(22.00) \\
\end{array}$ & $\begin{array}{c}\mathrm{CO} \\
\mathrm{C}_{7} \mathrm{H}_{4} \mathrm{~N}_{2} \\
\mathrm{C}_{2} \mathrm{H}_{2}\end{array}$ & $\begin{array}{c}{\left[\mathrm{C}_{13} \mathrm{H}_{8} \mathrm{~N}_{2} \mathrm{~S}\right]^{+}} \\
224(29.00) \\
{\left[\mathrm{C}_{6} \mathrm{H}_{4} \mathrm{~S}\right]^{+}} \\
108(100) \\
{\left[\mathrm{C}_{4} \mathrm{H}_{2} \mathrm{~S}\right]^{+}} \\
82(16.80)\end{array}$ & $\begin{array}{c}253\left(\mathrm{M}^{+}+1,13.8\right) 135(6.7), \\
134(16.5), 126(9.8), \\
112(7.1), 111(21.5), 91(7.4) \\
89(8.8), 88(10.4), 83(9.1), \\
75(16.2), 64(29.0), 63(36.7), \\
62(34.3), 61(11.8), 58 \\
(35.4), 57(15.8), 52(16.8), \\
51(24.9) .\end{array}$ \\
\hline
\end{tabular}




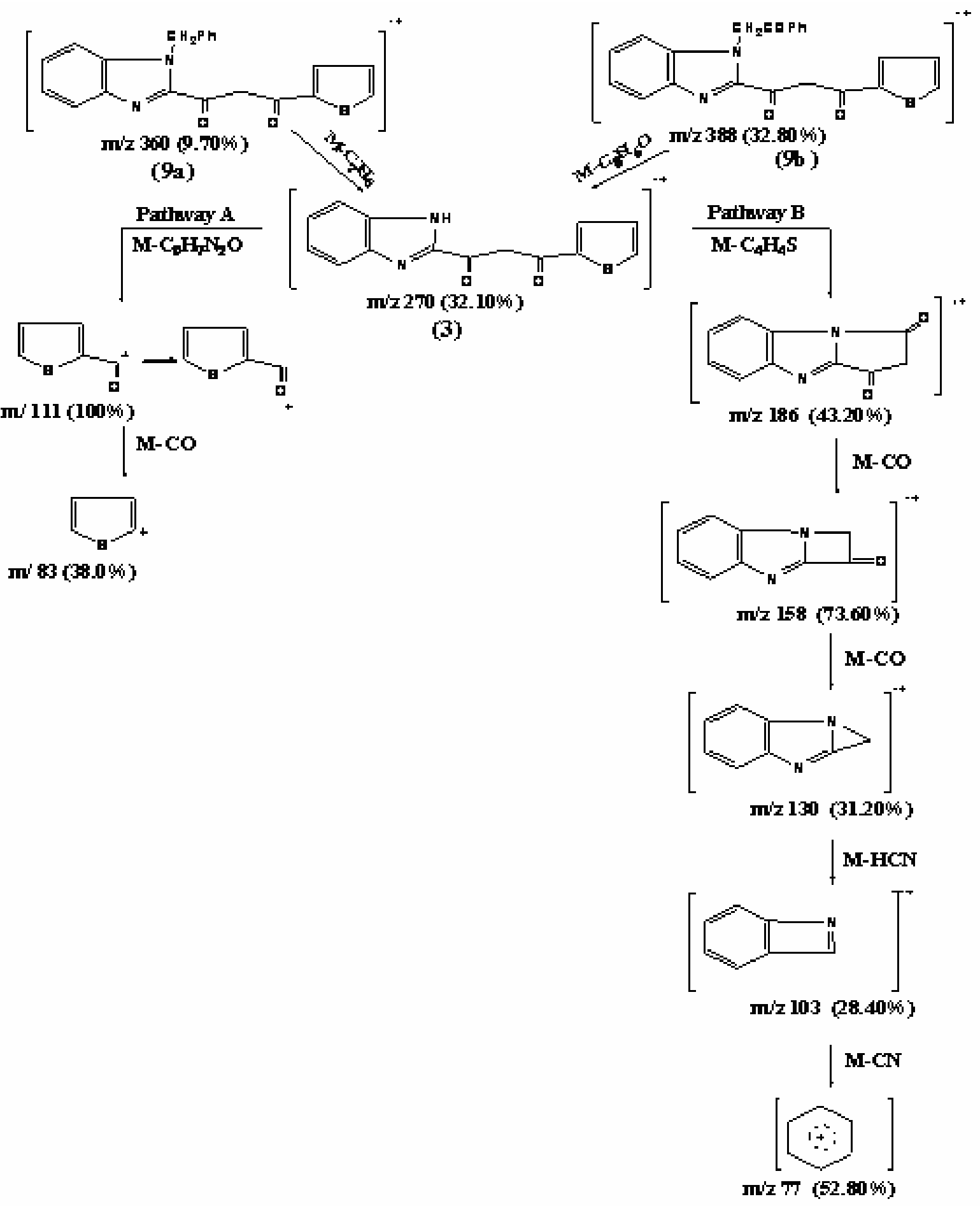

Scheme 6. Mass fragmentation pattern of compounds 3. $9 \mathrm{a}$ and $9 \mathrm{~b}$

The molecular ion of compound $\mathbf{1 0}$ at $\mathrm{m} / \mathrm{z}$ 294 fragmented further and involved two different possible pathways, as illustrated by Scheme 7 (Fig 7). The molecular ion of compound 11 fragmented and involved two pathways as illustrated in Table 2 (Fig. 8).
The electron impact ionization mass spectra of compounds 10 and 11 show a base peak of $\mathrm{m} / \mathrm{z}$ 220 and $\mathrm{m} / \mathrm{z} 108$, while the base peak of compounds $\mathbf{2 b}, \mathbf{3}, \mathbf{4} \mathbf{a}$ and $\mathbf{9 b}$ is the ion of $m / z 111$. The mass spectra of compound 5 show a base peak of $m / z 305$, while the base peak of compound 9a is the molecular ion of compound $\mathbf{3}$ of $\mathrm{m} / \mathrm{z} 270$. 


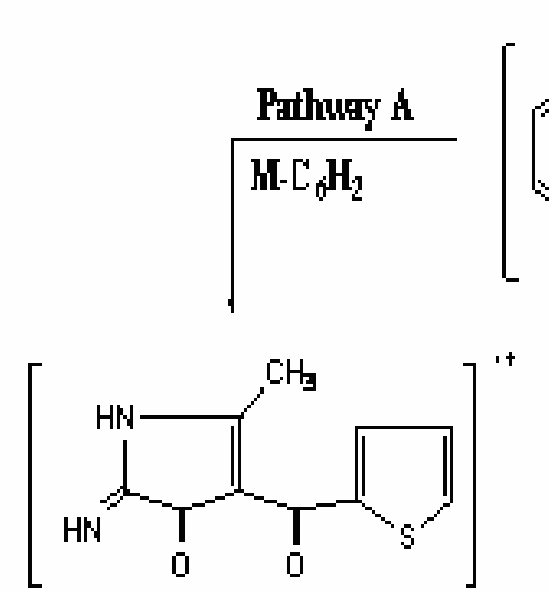

$\pi / 2230(100 \%)$

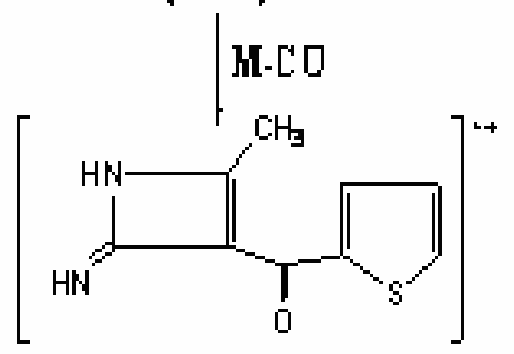

mi $192(860 \%)$

M.HCW

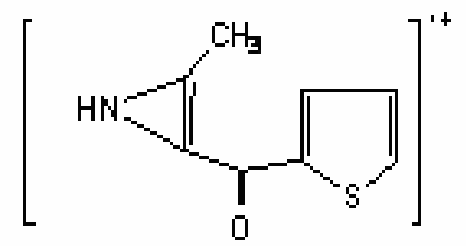

$\mathrm{m} / \mathrm{s} 165(7.70 \%)$

M. $\mathrm{C}_{3} \mathrm{H}_{4} \mathrm{~N}$<smiles>O=Cc1ccc([I+](=O)c2cccs2)s1</smiles>

mis $1116120 \%$

M.C.<smiles>c1ccsc1</smiles>

$m / 2836.60 \%)$

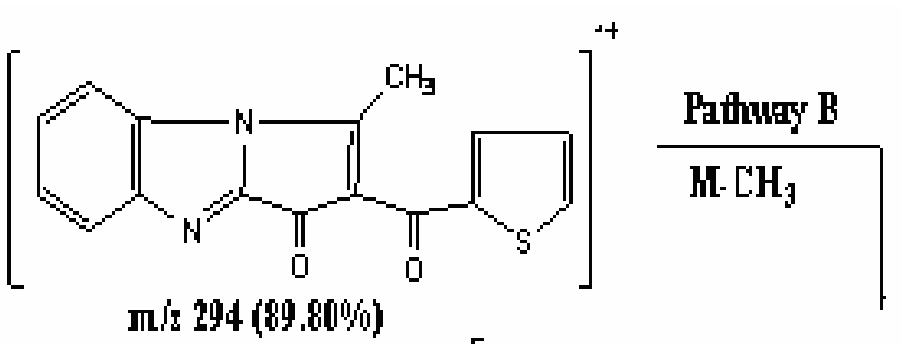<smiles>OC(C1=[C]n2c1nc1ccccc12)c1cccs1</smiles>

$\mathrm{m} / 279(89.80 \%)$

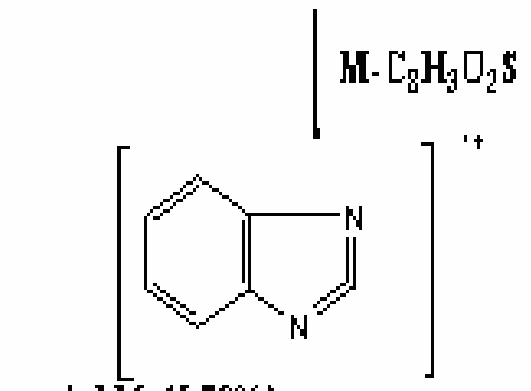

mis $116(5.70 \%)$

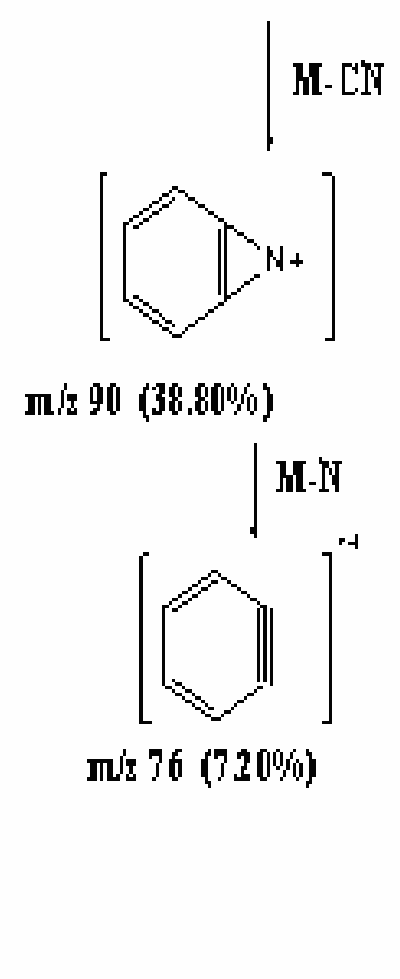

Scheme 7. Mass fragmentation pattern of compound 10 


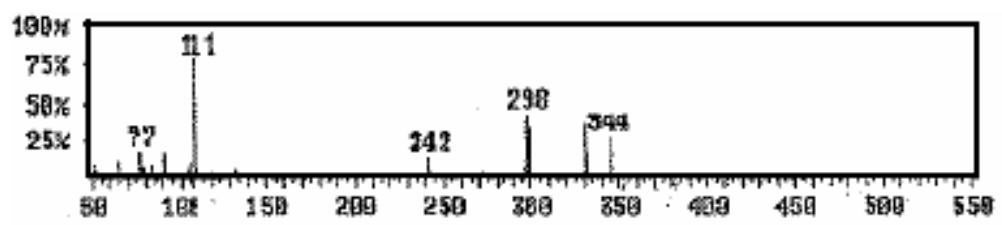

Fig. 1. Mass fragmentation of compound $2 b$

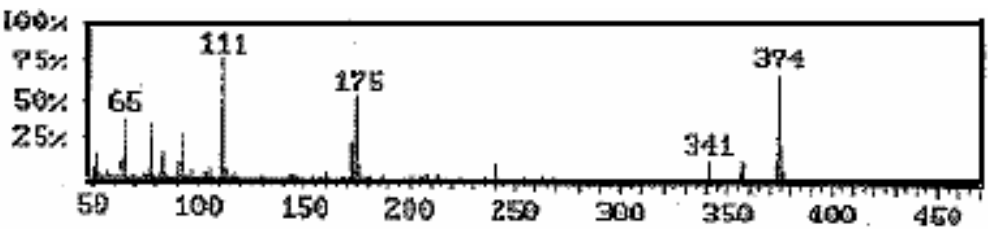

Fig. 2. Mass fragmentation of compound $4 a$

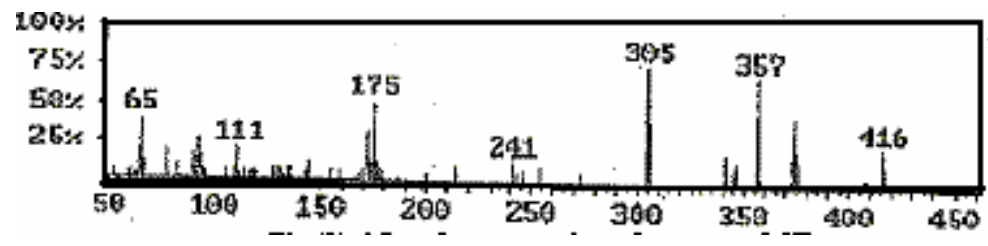

Fig. 3. Mass fragmentation of compound 5

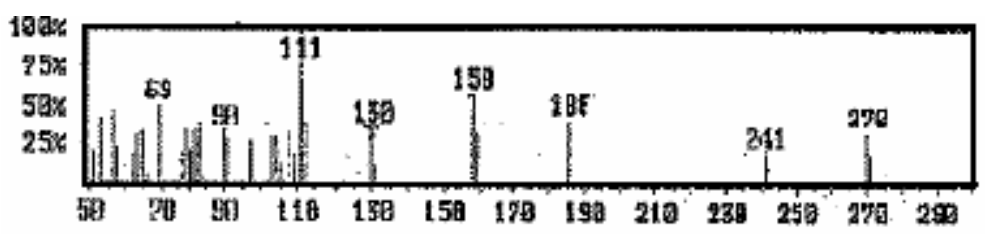

Fig. 4. Mass fragmentation of compound 3

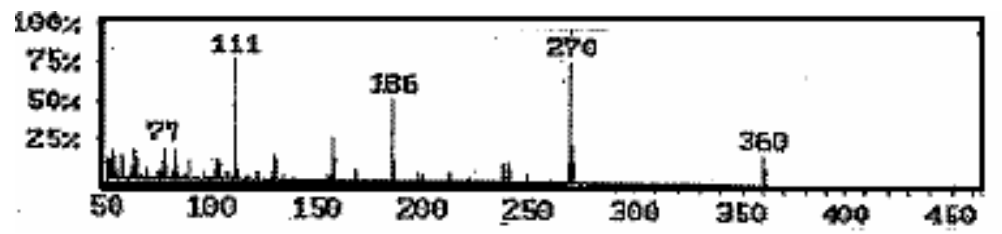

Fig. 5. Mass fragmentation of compound $9 \mathrm{a}$

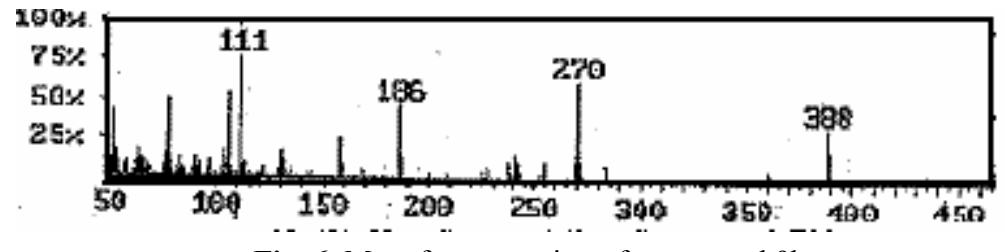

Fig. 6. Mass fragmentation of compound $9 \mathrm{~b}$

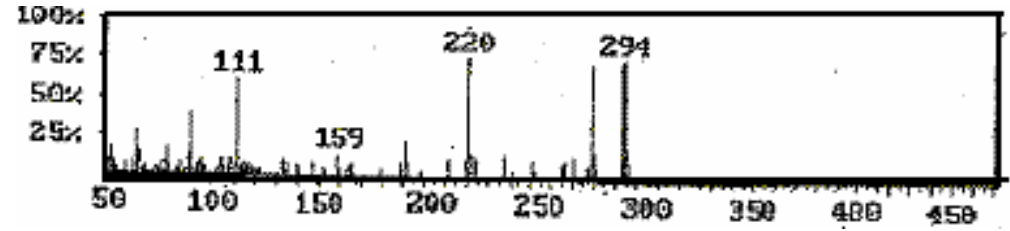

Fig. 7. Mass fragmentation of compound 10

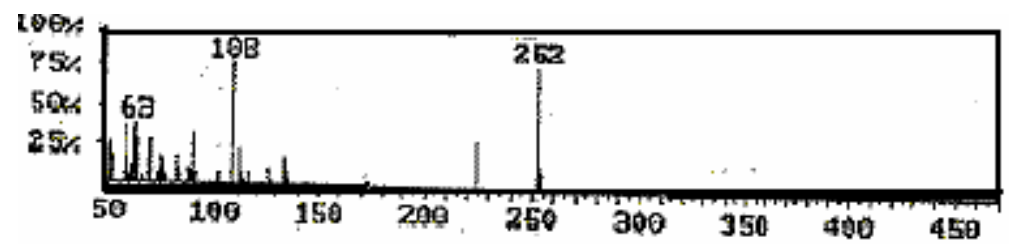

Fig. 8. Mass fragmentation of compound 11 


\section{REFERENCES}

[1] M. Tobbe, W. Spitzer, F. Victor, S. Miller, C. Lee, T. Sattelberg, E. Mckinney, C. Tang, Antirhino/Enteroviral Vinylacetylene Benzimidazoles: A Study of Their Activity and Oral Plasma Levels in Mice, J. Med. Chem., 40, 3937-3946 (1997).

[2] A. Porcari, R. Devivar, L. Kučera, J. Drach, L. Townsend, Design, Synthesis and Antiviral Evaluations of 1(Substituted benzyl)-2-Substituted-5,6-dichlorobenzimidazoles as Nonnucleoside Analogues of 2,5,6-Trichloro1-( $\beta$-D-ribofuranosyl)benzimidazole, J. Med. Chem., 41, 1252-1262 (1998).

[3] M. Roth, M. Morningstar, P. Boyer, S. Hughes, R. Bukheit, C. Michejda, Synthesis and Biological Activity of Novel Nonnucleoside Inhibitors of HIV-1 Reverse Transcriptase. 2-Aryl-Substituted Benzimidazoles, J. Med. Chem., 40, 4199-4207 (1997).

[4] M. Mrgawa, J. Girardet, J. Walker, G. Koszalka, S. Chamberlain, J. Drach, L.Townsend, Design, Synthesis, and Antiviral Activity of $\alpha$-Nucleosides: D- and L-Isomers of Lyxofuranosyl and (5-Deoxylyxofuranosyl)benzimidazoles, J. Med. Chem., 41(8), 1242-1251 (1998).

[5] I. Tamm, P. Sehgal, Halobenzimidazole Ribosides and RNA Synthesis of Cells and Viruses. Adv. Virus Res., 22, 187-192 (1979).

[6] I. Tamm, Inhibition of Influenza and Mumps Virus Multiplication by 4,5,6- (or 5,6,7-) Trichloro-1- $\beta$-D-Ribofuranosylbenzimidazole, Science, 120, 847-848 (1954).

[7] Y. Bai, J. Lu, Z. Shi, B. Yang, Synthesis of 2,15-Hexadecanedione as a Precursor of Muscone, Syn. Lett., 544-546 (2001).
[8] B. Sazen, D. Sames, Cobalt-Catalyzed Arylation of Azole Heteroarenes via Direct C-H Bond Functionalization, Org. Lett., 5(20), 3607-3610 (2003).

[9] J. Lewis, S. Wiedenam, R. Bergman, J. Elman, Arylation of Heterocycles via Rhodium-Catalyzed C-H Bond Functionalization, Org. Lett., 6, 35-38 (2004).

[10] H. Matsushita, S. Lee, M. Joung, B. Chapham, K. Janda, Smart Cleavage Reactions: The Synthesis of Benzimidazoles and Benzothiazoles from Polymer-bound Esters, Tetrahedron Lett., 45, 313-316 (2004).

[11] R. Saleh, I. El-Deen, Synthesis and Reactions of Ethyl 2Furoylpyruvate, J. Serb. Chem. Soc., 56(10), 595-600 (1991).

[12] I. El-Deen and F. Mohamoud, Synthesis and Chemical Behavior of 2-Chloro-3-(2'-thenoylmethyl) Quinoxaline Towards Some Nucleophilic Reagents, Phosporus, Sulfur Silicon Relat. Elem , 165, 205-212 (2000).

[13] R. Saleh, Synthesis of Butyl 2(H)-oxonaphtho[1,2-b]pyran-4-acetate and Butyl 3(H)-oxonaphtho[2,1-b]pyran-1acetate and Their Reactions, J. Indian Chem., 29B (3), 239-243 (1990).

[14] A. Farag, M. Al-Gharib, Synthesis and Reactions of C-(2Thienoyl)- $N$-arylformhydrazidoyl Bromides, Org. Prep. Froc. In., 20(5), 521-526 (1988).

[15] A. Farag, H. Hasaneen, I. Abbas, A. Shawali, M. Algharib, Synthesis and Reactions of Some 2-Thienyl- and 2-Thenoyl-derivativies of Thiazole and Thiadiazoline and Their Selenium Analogs, Phosphorus Sulfur and Silicon, 40, 243-249 (1988). 